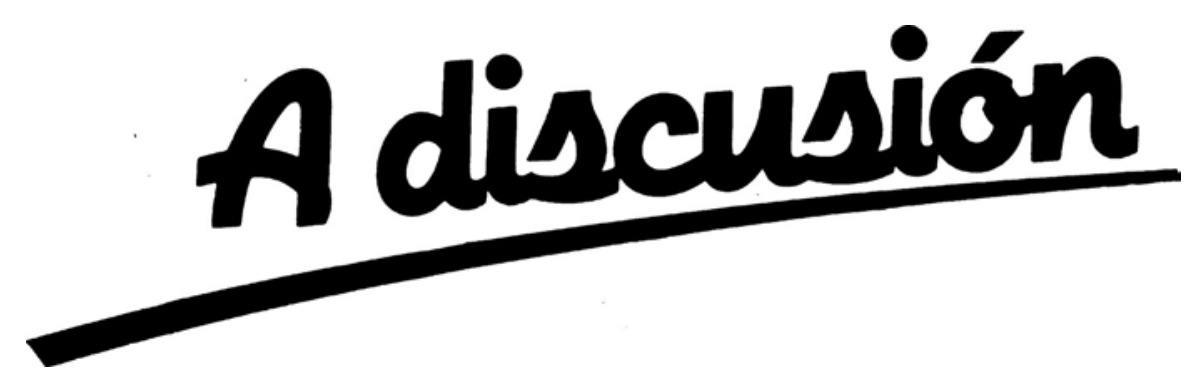

MODELING THE EURO OVERNIGHT RATE* Francis Benito, Ángel León and Juan Nave ${ }^{* *}$

WP-AD 2006-11

Correspondence: Ángel León. Dpto. Economía Financiera, Contabilidad y Marketing. Facultad de Ciencias Económicas. Universidad de Alicante. Apartado de correos 99, 03080 Alicante, Spain. E-mail: aleon@ua.es

Editor: Instituto Valenciano de Investigaciones Económicas, S.A.

Primera Edición Junio 2006

Depósito Legal: V-2382-2006

IVIE working papers offer in advance the results of economic research under way in order to encourage a discussion process before sending them to scientific journals for their final publication.

\footnotetext{
* We are grateful to Sunil Mohanty, Eliseo Navarro and Alfonso Novales for helpful suggestions, as well as seminar audiences at Multinational Finance Society (Athens, 2005), the Meeting on Financial Mathematics (Verbania, 2005), the Meeting of Applied Economy (Murcia, 2005) and the Finance Forum (Madrid, 2005). Of course, the usual caveat applies. Corresponding author: Angel León, Dpto. Economía Financiera, Facultad de Ciencias Económicas, Universidad de Alicante, Apartado de Correos 99, 03080 Alicante, Spain; E-mail: aleon@ua.es

** Francis Benito: University of Alicante, Ángel León: University of Alicante and Juan Nave: University of Castilla La Mancha.
} 


\title{
MODELING THE EURO OVERNIGHT RATE
}

\author{
Francis Benito, Ángel León and Juan Nave
}

\begin{abstract}
This paper describes the evolution of the daily Euro overnight interest rate (EONIA) by using several models containing the jump component such as a single regime ARCH-Poisson-Gaussian process, with either a piecewise function or an autoregressive conditional specification (ARJI) for the jump intensity, and a two regime-switching process with jumps and time varying transition probabilities. To model the jump intensity, we include the following effects which are significant for the occurrence of jumps such as: (1) the end of maintenance period effect because of reserve requirements, (2) the end of month effect, also known as the calendar day effect, caused mainly by the accounting adjustments and finally, (3) the meeting effect caused by the fortnightly meetings of the Governing Council of the European Central Bank (ECB). These effects lead to a better performance and several of them are also included for the behavior of the transition probabilities. Since the target of the ECB is keeping the EONIA rate close to the official rate, we have modeled the conditional mean of the overnight rate series as a reversion process to the official rate distinguishing two alternative speeds of reversion, in concrete, a different speed if EONIA is higher or lower than the official rate. We also study the jumps of the EONIA rate around the ECB's meetings by using the ex-post probabilities of the ARJI model. Finally, we develop an out-of-sample forecasting analysis to measure the performance of the different candidate models.
\end{abstract}

JEL classification: C13, C22, E43, E52.

Keywords: ARCH-Poisson-Gaussian; Regime switching; mean reversion; Autoregressive conditional jump intensity; Maintenance period; Calendar day effect; ECB's meeting. 


\section{Introduction}

The control of very short-term interest rates, specifically the interest rate of the interbank market for unsecured overnight loans - the EONIA ${ }^{1}$ for the Eurozone - is the first step in the mechanism for the monetary policy transmission and the basis on which it acts in order to influence the behavior of longer term interest rates. Accordingly, the central banks are very interested in understanding its dynamics and how it relates to monetary policy decisions.

The subject of several papers in recent years is about understanding the empirical characteristics of the EONIA rate and how effective monetary policy measures are in order to keep its evolution close to the official rate. However, the literature has not yet covered many of the aspects analysed in other interest rate markets. For example, in the United States the analysis of Federal Funds is much wider than the study of EONIA in the Eurozone. The earliest studies carried out on the empirical characteristics of the Federal Funds include the works by Hamilton (1996) and Balduzzi et al. (1997), while one of the most recent is the work of Bartolini et al. (2002).

In the Eurozone most works have concentrated on knowing whether both instruments and procedures to implement the monetary policy have a repercussion on the overnight rate. Thus, for example, it has been analysed the implications of the institutional details of the reserve market on the behavior of the EONIA and how this rate is affected by the liquidity management of the European Central Bank (ECB). In this regard, Hartmann et al. (2001) and Manna et al. (2001) concentrate on analysing the operational framework of ECB, while either Bindseil and Seitz (2001) or Angeloni and Bisagni (2002) concentrate more on the consequences of the liquidity conditions. Prati et al. (2002) carried out a study on how the operational procedures and intervention forms of the central banks affect the characteristics and behavior of the one-day rate in the most industrialised countries (Eurozone and G7). Gaspar et al. (2001) drew up a model for the behavior of the overnight rate during the reserve maintenance period, mainly in order to estimate the impact of the announcement of monetary policy measures. Würtz (2003) carried out a descriptive model 
aimed at an exhaustive list of all the characteristics and variables that may explain the behavior of the EONIA rate. Moschitz (2004a) modelled the problem of the intertemporal decision in the reserve market, both for the central bank and for commercial banks. Pérez Quirós and Rodríguez Mendizábal (2005) checked whether there are statistical differences in the behavior of the daily rate before and after the European Monetary Union (EMU), presenting a model for liquidity shocks focused from the demand side. Other works by Moschitz (2004b), Blanco (2005) and Cassola and Morana (2003, 2004), among others, focus on understanding the volatility transmission from the EONIA rate along the yield curve.

Many of these authors reach common conclusions with respect to the behavior of the overnight rate. They are unanimous regarding the martingale hypothesis does not hold. One widely tested empirical characteristic is the existence of predictable patterns in the behavior of the EONIA rate. The most obvious non-compliance with the hypothesis come from the effect known as the end of maintenance period effect and the calendar effects (end of month, quarter, half-year and year). The former is a consequence of the averaging provisions to comply with reserve requirements, while the calendar effects may be attributed to the window-dressing activities performed by banks on their balances when they must be presented. Together with these effects, the periodical meetings that the Governing Council of the ECB holds fortnightly to assessment the Eurosystem's monetary policy, constitute the main causes of the jumps observed in the dynamics of the EONIA rate.

In this context, our aim is to tackle the evolution of the EONIA rate through a jump diffusion model which covers these three effects. As starting model, we will use the Poisson-Gaussian process with ARCH conditional variance implemented by Das (2002) for modeling the federal funds rate. This model, in addition to capturing properly the empirical characteristics observed in the EONIA series, allows us to know the jump intensity caused by each of the three effects studied. Likewise, and given that the aim of the ECB is to maintain the EONIA rate close to the official rate, we will model the conditional mean of this overnight interest rate as a reversion process to this official rate,

\footnotetext{
1 The EONIA (Euro OverNight Index Average) is published by the European Banking Federation as the balanced average of all overnight unsecured lending transactions between the most active credit institutions in the Eurozone money markets.
} 
distinguishing two alternative speeds of reversion, in concrete, a different speed if EONIA is higher or lower than the official rate. We will test the existence of market overreaction as another cause of the violation of the martingale property. An extension of the above model is also implemented here, specifically the autoregressive conditional jump intensity model by Chan and Maheu (2002). This model will also be used to analyse the behavior of jumps around the days of the ECB's meetings through the ex-post probabilities implicit in this model. Finally, we develop a two-regime switching model with time varying transition probabilities and each regime is characterised by an ARCH-Poisson-Gaussian process. The regime switching model outperforms simple single-regime models for interest rates as many empirical applications confirm such as Gray (1996) and so forth.

The rest of this paper is divided into nine sections. The next section describes the effects mentioned above. Section three analyses EONIA rate data corresponding to the period under analysis. Section four specifies the first model used here that includes a study of the market overreaction. Section five shows the second model, results and the study of jumps around the ECB's meetings. Section six exhibits the regime switching process and results. Section seven shows both an in-sample and out-of-sample forecasting analysis for the different models and finally, section eight concludes.

\section{Stylized facts of EONIA behavior}

In order to meet its aims, including the control of very short-term interest rates, the Eurosystem uses a series of instruments and procedures which make up its operational framework, specifically: it carries out open market operations, it offers standing facilities and it demands that credit institutions hold minimum reserves.

The most important open market operations are the main refinancing operations $(\mathrm{MRO})^{2}$. The interest rate from these operations represents the guideline of the monetary policy. The Eurosystem also offers standing facilities to its counterparty institutions, who may recur on their own initiative to the marginal lending facility (overnight liquidity from

\footnotetext{
${ }^{2}$ The MRO represent the main injection of liquidity by the ECB into the financial system, mainly through regular auctions. Until 28 June 2000 the intervention interest rate matched the interest rate applied to fixed rate auctions. From that moment it has coincided with the minimum bid rate for variable interest rate auctions.
} 
national central banks at a pre-specified interest rate against eligible assets); and the deposit facility (overnight deposits with national central banks at a pre-specified interest rate). The interest rates applied to these facilities are, in general, unfavourable as regards market interest rates, which determine the fluctuation band of the EONIA rate with the aim of reducing the volatility. In Figure 1, together with the EONIA rate, we can see the evolution of the rates of the MRO, marginal lending facility and deposit facility, for the period under study.

Finally, the ECB requires credit institutions to hold minimum reserves on accounts with the national central banks. The compliance with such reserve requirements may lead to a mechanism of averages, that is, the minimum reserves to maintain are calculated as an averaging provision for the whole month covered by the maintenance period, not for each day. The maintenance period begins on the $24^{\text {th }}$ calendar day of each month and ends on the $23^{\text {rd }}$ calendar day of the following month ${ }^{3}$. The ECB uses this average mechanism to stabilize interest rates. Credit institutions may soften daily liquidity fluctuations, as the transitory reserve imbalances may be compensated with imbalances in the other direction within the same maintenance period. This averaging provision allows for a certain intertemporal arbitrage ${ }^{4}$ which should, in theory, guarantee equality throughout the maintenance period between the current level and the expected level of the shortest interest rate at the end of the maintenance period. However, towards the end of the maintenance period, and especially after the last MRO, the reserve must be adapted to comply with the required minimum average. It is not possible to transfer a deficit or surplus liquidity into the future, neither turn to an MRO. This leads to the peaks observed in the series of the EONIA rate towards the end of the maintenance period.

The calendar effects are due to the window dressing activities to which banks adjust their balances at the end of the month, making the demand for one-day funds shoot up. As

\footnotetext{
${ }^{3}$ From March 8, 2004 the schedule for reserve maintenance periods always starts on the settlement day of the first main financing operation (MRO) following the Governing Council meeting at which the monthly assessment of the monetary policy stance is prescheduled and will end on the day preceding the corresponding settlement day in the following month (see "The monetary policy of the ECB" in year 2004 available on http://www.ecb.int). We do not study the effect of this new schedule of the maintenance period on the EONIA behavior due to the lack of enough data.

${ }^{4}$ The mechanism allows credit institutions to grant loans on the market (and incur in reserve deficits) when the shortest term money market interest rates are above the expected levels for the rest of the maintenance period. Otherwise, they may take loans on the market and maintain a surplus of reserves.
} 
Moschitz (2004a) says, the central banks cannot do much to cancel these effects, as changes in the offer of reserves during these days would be interpreted as temporal changes and, therefore, would not affect the behavior of the interest rate.

The final effect considered is the effect caused by the fortnightly meetings of the Governing Council of the $\mathrm{ECB}^{5}$, after which changes of the rate of the MRO may occur. The expectation regarding changes in the official rate is one of the important variables in the behavior of the EONIA rate. One of the concerns of the central banks is to avoid an increase of the volatility when the official rate is modified. The aim is to communicate monetary policy measures clearly and not to give confusing signals to the market, especially when expectations regarding interest rates vary in the middle of a maintenance period. This aspect is studied by Gaspar et al. (2001) who, using a time series going from January 1999 to March 2001, come to the conclusion that the market anticipates monetary policy changes, so that the announcement of a new rate does not cause important changes either in the level or volatility of overnight rates. However Moschitz (2004a), using a wider series up to February 2004, found that banks react slowly to changes in the official rate, with an increased volatility around the day of the announcement of the monetary policy decision.

\section{Data}

This section analyses the main characteristics of the EONIA interest rate for a sample of 1303 daily observations over the period from January 1999 - starting date of Stage Three of the EMU - up to December 2003. As Figure 1 shows the EONIA rate has, in general, been close to the rate of the MRO, which shows the importance of these operations pursuing the aims of steering interest rates. The fluctuations in the overnight rate, which can be seen in the graph, reflect liquidity conditions that are temporarily relaxed or restrictive on the money market. As we have already said, these fluctuations and the peaks are related mainly to the aforementioned averaging provisions for the reserve

\footnotetext{
${ }^{5}$ The Governing Council meets twice a month, on alternative Thursdays. At its first meeting, as a rule, the Governing Council assesses the economic situation and the stance of the monetary policy. Decisions on the key interest rates are normally taken during that meeting. At its second meeting, the Governing Council focuses on issues related to other tasks and responsibilities of the ECB and the Eurosystem. Obviously, if warranted by the circumstances, the Governing Council can still decide to change the key ECB interest rates at any time, regardless of previously scheduled meetings.
} 
requirements, but also to the calendar effects and the fortnightly meetings of the Governing Council of the ECB.

Figure 1. Evolution of the following rates: EONIA, MRO, Marginal lending facility and Deposit facility for the period from 4 January 1999 to 31 December 2003.

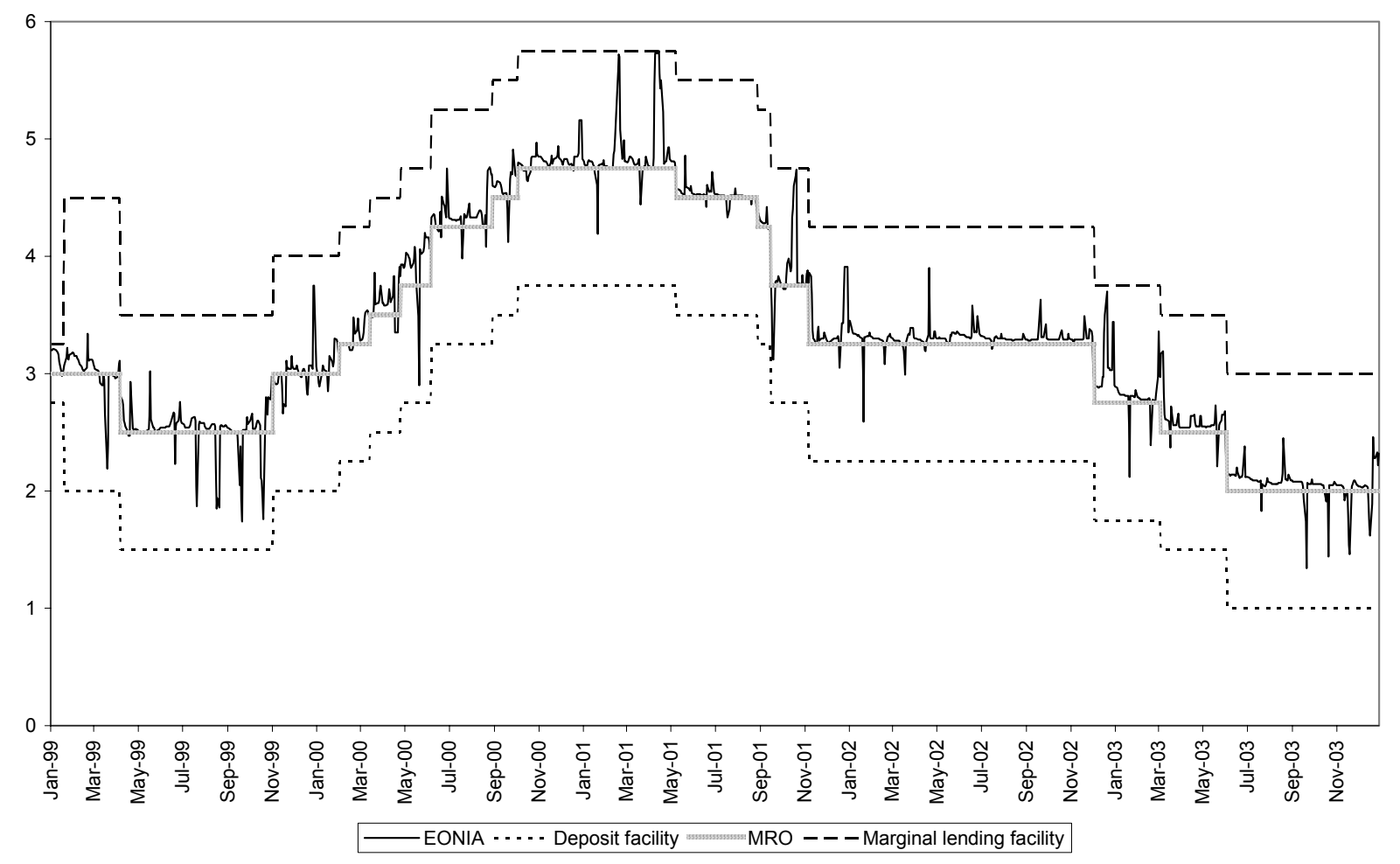

Table 1 shows the descriptive statistics for the EONIA rate and its first difference. The maximum value (5.75\%) was reached on 17 April 2001, and the minimum (1.34\%) on 23 September 2003. The largest rise (1.16\%) occurred on 24 May 2000 and the largest drop $(0.98 \%)$ on 24 October 2001 . We can see that both of them occurred on day 24 of the month, precisely the first day of each reserve maintenance period, when the EONIA recovers the value that it reached on the days before the end of the reserve maintenance period. Moreover, the Jarque-Bera statistic rejects normality for both the series: level $\left(r_{t}\right)$ and first difference $\left(\Delta r_{t}\right)$. Note that $\Delta r_{t}$ shows a high level of asymmetry and leptokurtosis. 
Table 1. Descriptive statistics

\begin{tabular}{lcc}
\hline \hline & $\begin{array}{c}\text { EONIA rate } \\
(r)\end{array}$ & $\begin{array}{c}\text { First difference of } \\
\text { EONIA rate } \\
(\Delta r)\end{array}$ \\
\hline \hline Mean & 3.3740 & -0.0006 \\
Median & 3.2900 & 0.0000 \\
Maximum & 5.7500 & 1.1600 \\
Minimum & 1.3400 & -0.9800 \\
Std. Dev. & 0.9021 & 0.1417 \\
Skewness & 0.3199 & 0.8631 \\
Kurtosis & 2.1739 & 16.8901 \\
\hline \hline Jarque-Bera & 59.2737 & 10628.38 \\
(p-value) & 0.0000 & 0.0000 \\
\hline \hline
\end{tabular}

Descriptive statistics of the daily rate and its first difference over the period January 1999 to December 2003. The data are in percentage terms.

Table 2 provides the high persistence that the EONIA series exhibits by looking at the sample autocorrelation coefficients. Note the high values they show and how slowly they tend to decrease. This suggests evidence of the possible presence of a unit root, as ratified by the Augmented Dickey-Fuller (ADF) test. This test, however, rejects the presence of a unit root for the series of $\Delta r_{t}$. Finally, note that the correlation coefficients of $\Delta r_{t}$ are small and negative. This behavior suggests a pattern of mean reversion.

\section{ARCH-Poisson-Gaussian model}

A Poisson-Gaussian process with ARCH volatility is selected for modeling the dynamics of the change in the EONIA rate or $\Delta r_{t}$. This process results to be suitable for the empirical characteristics of the series under study. As the model allow for jumps, we can perfectly capture the peaks observed in the overnight rate series and also the high level of leptokurtosis, which is shown in Table 1, makes the use of these models very appropriate. On the other hand, empirical evidence shows that jump models diminish the possible no linearity of the conditional mean of the series. As regards the behaviour of the 
Table 2. Autocorrelation coefficients and unit root test

\begin{tabular}{ccc}
\hline \hline & $\begin{array}{c}\text { EONIA rate } \\
(r)\end{array}$ & $\begin{array}{c}\text { First difference of } \\
\text { EONIA rate } \\
(\Delta r)\end{array}$ \\
\hline \hline$\rho_{1}$ & 0.987 & -0.106 \\
$\rho_{2}$ & 0.977 & -0.152 \\
$\rho_{3}$ & 0.970 & -0.055 \\
$\rho_{4}$ & 0.965 & -0.054 \\
$\rho_{5}$ & 0.961 & -0.035 \\
$\rho_{6}$ & 0.958 & -0.034 \\
$\rho_{7}$ & 0.956 & 0.004 \\
\hline \hline ADF & -0.6785 & -21.4034 \\
Critical value & -1.6165 & -2.5667 \\
\hline \hline
\end{tabular}

ADF denotes the Augmented Dickey-Fuller unit root test statistic with a $10 \%$ critical value of -1.6165 for EONIA rate and with a $1 \%$ critical value of -2.566 for changes in the EONIA rate. The number of lags used is four.

interest rate volatility, it is widely accepted that these series have a high level of conditional heteroskedasticity in the variance. In this way, a GARCH family specification for volatility allows us to capture both the clustering effect and the high persistence intrinsic to $\Delta r_{t}$.

Let define the information set available at time $t$ to be the history of daily interest rates, both the overnight and the official rates, and denoted as $\Phi_{t}$. The model for the EONIA rate presents the following specification:

$$
\Delta r_{t}=\mu_{t}+\sigma_{t} z_{t}+J_{t} \Delta n_{t}
$$

where $\mu_{t}$ is the expected conditional mean, that will be characterised later, when there is no jump; $z_{t}$ is an independent standard normal variable; $J_{t}$ is the jump size that is also assumed normally and independent distributed with a time varying mean denoted as $\theta_{t}$ and variance $\psi^{2}(\psi>0)$, that is assumed to be constant in order to simplify the model. 
Likewise, we assume that $z_{t}$ and $J_{t}$ are also independent. Moreover $\Delta n_{t}$ designates the Poisson process with $\lambda_{t}$ as the time-varying intensity parameter for the number of jumps which occur in the interval from $t-1$ to $t$, specifically $(t-1, t]$. We approach the Poisson process with a Bernoulli distribution - see Das (2002) - conditioned to $\Phi_{t-1}$ with probability $\lambda_{t}$ when there is a jump, that is $P\left(\Delta n_{t}=1 \mid \Phi_{t-1}\right)=\lambda_{t}$, and so a probability $1-\lambda_{t}$ when there is no jump. Note that $\lambda_{t}$ is interpreted as the ex-ante probability of occurring one jump. We specify the conditional variance $\sigma_{t}^{2}$ with an $\mathrm{ARCH}$ (1) feature ${ }^{6}$ so as to limit the number of estimated parameters. Finally, it is important to mention that $\mu_{t}, \sigma_{t}, \theta_{t}$ and $\lambda_{t}$ are measurable with respect to the information set $\Phi_{t-1}$.

\subsection{Likelihood function and conditional moments}

The hypothesis underlying equation (1) implies that the distribution of $\Delta r_{t}$, conditioned to the most recent information set and to $j$ jumps, is normally distributed as

$$
f\left(\Delta r_{t} \mid \Delta n_{t}=j, \Phi_{t-1}\right)=\frac{1}{\sqrt{2 \pi\left(\sigma_{t}^{2}+j \psi^{2}\right)}} \exp \left(-\frac{\left(\Delta r_{t}-\mu_{t}-j \theta_{t}\right)^{2}}{2\left(\sigma_{t}^{2}+j \psi^{2}\right)}\right)
$$

where, for our purposes, $j$ takes either value 0 or 1 . The conditional density function of $\Delta r_{t}$ is obtained from

$$
f\left(\Delta r_{t} \mid \Phi_{t-1}\right)=\left(1-\lambda_{t}\right) \times f\left(\Delta r_{t} \mid \Delta n_{t}=0, \Phi_{t-1}\right)+\lambda_{t} \times f\left(\Delta r_{t} \mid \Delta n_{t}=1, \Phi_{t-1}\right)
$$

and its log-likelihood, denoted as $L$, is calculated as $\sum_{t=1}^{T} \ln f\left(\Delta r_{t} \mid \Phi_{t-1}\right)$ where $T$ indicates the size of the sample.

The conditional mean of (3) is

\footnotetext{
${ }^{6}$ The same volatility structure is imposed by Das (2002) for modeling the Fed Funds rate.
} 


$$
E_{t-1}\left(\Delta r_{t}\right)=\mu_{t}+\lambda_{t} \theta_{t}
$$

where $E_{t-1}(\cdot)$ indicates the expectation conditioned to the information set $\Phi_{t-1}$. The conditional variance is obtained as

$$
V_{t-1}\left(\Delta r_{t}\right)=\sigma_{t}^{2}+\lambda_{t}\left[\psi^{2}+\left(1-\lambda_{t}\right) \theta_{t}^{2}\right]
$$

It can be seen that this conditioned variance contains two components. A first component defined as

$$
\sigma_{t, J}^{2}=\lambda_{t}\left[\psi^{2}+\left(1-\lambda_{t}\right) \theta_{t}^{2}\right]
$$

is associated with the innovation of the jumps and secondly, $\sigma_{t}^{2}$ is modelled with the following ARCH (1) structure:

$$
\sigma_{t}^{2}=\omega_{0}+\omega_{1}\left[\Delta r_{t-1}-E_{t-2}\left(\Delta r_{t-1}\right)\right]^{2}
$$

where it can be seen that $\omega_{0}, \omega_{1}>0$. Observe that equation (7) includes the effects of both innovations - normal innovations and innovations due to jumps - in the square of its past innovations.

\subsection{The no-jump component in the conditional mean}

As we have already mentioned, because the central bank tries to keep the daily rate close to the official rate, see Figure 1, we have modelled the conditional mean $\mu_{t}$ as a process reverting to the MRO rate. This rate displays a discontinuous behavior due to the discrete interventions carried out by the ECB. Accordingly, the rate of the MRO will represent the long-term average to which the EONIA rate should revert. One relevant characteristic of our model is the specification of two different speeds of reversion: one for high rates if the 
EONIA rate is above the official rate, and another for low rates when the daily rate is below the official rate. In short, the behavior of $\mu_{t}$ is guided by:

$$
\mu_{t}=\alpha_{1}\left(r_{t-1}-r_{t-1}^{*}\right)^{+}+\alpha_{2}\left(r_{t-1}-r_{t-1}^{*}\right)^{-}
$$

where $r_{t}$ is the overnight rate; $r_{t}^{*}$ is the rate of the MRO or official rate; the expression $\left(r_{t-1}-r_{t-1}^{*}\right)^{+}$is equal to $\max \left(r_{t-1}-r_{t-1}^{*}, 0\right)$; while $\left(r_{t-1}-r_{t-1}^{*}\right)^{-}$is equal to $\min \left(0, r_{t-1}-r_{t-1}^{*}\right)$; and $\alpha_{1}$ and $\alpha_{2}$ are the reverting coefficients, which must be negative to ensure that reversion to the official rate exists. An asymmetric behavior will hold if it is verified that $\alpha_{1} \neq \alpha_{2}$.

Finally, respecting the mean of the jump size $\theta_{t}$, we will assume two alternative specifications: one with a constant average, that is $\theta_{t}=\theta$; and another a mean reverting to the official rate, $\theta_{t}=\gamma\left(r_{t-1}-r_{t-1}^{*}\right)$ verifying that $\gamma<0$ to guarantee mean reversion to the rate of the MRO.

\subsection{The jump intensity}

As we have mentioned above, we know that there are certain days on which the EONIA rate has a greater jump probability: the last days of the maintenance period, end of month, quarter, half-year and year, and the days close to the periodical meetings of the Governing Council of the ECB. Accordingly, we make the jump arrival intensity $\lambda_{t}$ of these days depending on dummy variables. In this way, we assume a piecewise dynamics for the specification of the jump intensity:

$$
\lambda_{t}=\delta_{0} D_{t}^{o}+\delta_{1} D_{t}^{m p}+\delta_{2} D_{t}^{m}+\delta_{3} D_{t}^{m e e t}+\delta_{4} D_{t}^{m e e t+m p}+\delta_{5} D_{t}^{m e e t+m}
$$

where $D_{t}$ designates a certain dummy variable: 
$D_{t}^{m p}=1 ; \quad$ if the last four days of the maintenance period.

$D_{t}^{m}=1 ; \quad$ if the last two days of each month ${ }^{7}$.

$D_{t}^{\text {meet }}=1 ; \quad$ if day of meeting of the Governing Council of the ECB or next day.

$D_{t}^{\text {meet }+m p}=1$; if either the day of the meeting of the Governing Council of the ECB or the following day coincide with the last four days of a maintenance period.

$D_{t}^{\text {meet+m }}=1$; if either the day of the meeting of the Governing Council of the ECB or the following day coincide with the last two days of the month.

$D_{t}^{o}=1 ; \quad$ if any other day.

The aim of the dummy variables $D_{t}^{\text {meet+mp }}$ and $D_{t}^{\text {meet+m }}$ is to capture, and so to eliminate from the other dummy variables, those days where the effects analysed (end of maintenance period, end of month and meeting) coincide. Note that it could be impossible to distinguish which part of the jump intensity is due to which effect without the introduction of these variables. Summing up, it holds that

$$
D_{t}^{m p} \cap D_{t}^{m} \cap D_{t}^{\text {meet }} \cap D_{t}^{\text {meet }+m p} \cap D_{t}^{\text {meet }+m} \cap D_{t}^{o}=\varnothing .
$$

The choice of the last four days of the maintenance period and the last two days of each month was made after repeating the analysis with different combinations and observing that the best fit, under the Schwarz Information Criterion (SIC), was obtained with the combination above.

As observed by Pérez-Quirós and Rodríguez Mendizábal (2005), there is no consensus on how many days are affected by the end of the maintenance period effect. However, their theoretical model and much of the literature agree that the liquidity conditions play an important role after the last MRO of the maintenance period. This is due to the fact that no liquidity imbalance occurs after the last MRO of the maintenance period can be cleared by the ECB. In a simple graphic analysis, we have observed that it is necessary to eliminate at least four days in order to make disappear the peaks in the

\footnotetext{
${ }^{7}$ In order to reduce the number of dummy variables, we have grouped the end of month, quarter, half-year and year effects into a single end of month effect.
} 
EONIA series. Since the time between the last MRO of the maintenance period and the end of that period is one week at most, then four days would be a reasonable value.

With respect to the effect caused by the meetings of the Governing Council of the $\mathrm{ECB}$, we looked at the jump intensity occurring on the day of the meeting and on the following day. The announcement of the result of the meeting, with the possible modification of the official rate, is not known until 1:45 p.m. Indeed, the EONIA rate corresponding to the day of the meeting incorporates transactions made previously to such announcement and so it does not take into account the outcome of the meeting. All transactions carried out on the following day include the result of the meeting. In case of approving a change of the official rate, it comes into effect just on the next day after the meeting.

Finally, those effects that might cause jumps on other days are measured through the constant $\delta_{0}$.

\subsection{Results}

Tables 3 and 4 show the results obtained by the Poisson-Gaussian model with ARCH variance - under the two alternative specifications of both $\mu_{t}$ and $\theta_{t}$ - with a constant intensity parameter $\lambda$ and a time varying intensity $\lambda_{t}$ defined in equation (9) respectively. We can appreciate some features. First, there is mean reversion for both the single and double mean reversion processes, though the SIC value for the double mean reversion is higher and hence, a more adequate model. It is also verified that those models in Table 4 with a mean reversion structure for $\theta_{t}$ score a higher SIC value than the case of $\theta_{t}$ to be constant.

Second, if we concentrate on the parameters of the double mean reversion - see model 4 from Table 3 and models iii and $i v$ from Table 4 - under any specification for either the intensity parameter or the mean of the jump size, we observe two main characteristics: (1) the value of the parameters $\alpha_{1}$ and $\alpha_{2}$ are both negative and significant, confirming the reversion to the MRO interest rate; and (2) this reversion is 
asymmetric since $\alpha_{1}$ and $\alpha_{2}$ are different. Because of $\left|\alpha_{1}\right|$ is lower than $\left|\alpha_{2}\right|$, it also holds that the speed of reversion for low rates (and lower than the official rate) is greater than the speed of reversion for high rates (and higher than the official rate). So, the EONIA rate reverts to the official rate more quickly from drops than from rises.

Table 3 ARCH-Gaussian and ARCH-Poisson-Gaussian process with constant intensity parameter $\lambda$

Daily data of EONIA rate covering the period January 1999 to December 2003. This table contains the ARCHGaussian process (no jumps) and the ARCH-Poisson-Gaussian process under different features of $\mu_{t}$. The ARCHPoisson-Gaussian process with a constant intensity parameter $\lambda$ is specified as follows:

$$
\Delta r_{t}=\mu_{t}+\sigma_{t} z_{t}+J_{t} \Delta n_{t} ; \quad z_{t} \sim N(0,1) ; J_{t} \sim N\left(\theta_{t}, \psi^{2}\right) ; z_{t} \text { and } J_{t} \text { are independent }
$$

where $\theta_{t}=\gamma\left(r_{t-1}-r_{t-1}^{*}\right) ; \sigma_{t}^{2}=\omega_{0}+\omega_{1}\left[\Delta r_{t-1}-E_{t-2}\left(\Delta r_{t-1}\right)\right]^{2}$ and $\mu_{t}$ is defined in the table below.

\begin{tabular}{|c|c|c|c|c|}
\hline & \multicolumn{2}{|c|}{$\mu_{t}=\alpha\left(r_{t-1}-r_{t-1}^{*}\right)$} & \multicolumn{2}{|c|}{$\mu_{t}=\alpha_{1}\left(r_{t-1}-r_{t-1}^{*}\right)^{+}+\alpha_{2}\left(r_{t-1}-r_{t-1}^{*}\right)^{-}$} \\
\hline & Model 1 & Model 2 & Model 3 & Model 4 \\
\hline Parameters & ARCH-Gaussian & $\begin{array}{l}\text { ARCH-Poisson- } \\
\text { Gaussian }\end{array}$ & ARCH-Gaussian & $\begin{array}{l}\text { ARCH-Poisson- } \\
\text { Gaussian }\end{array}$ \\
\hline$\alpha$ & $\begin{array}{c}-0.173 \\
(-5.084) \\
\end{array}$ & $\begin{array}{l}-0.0317 \\
(-4.542) \\
\end{array}$ & --- & -- \\
\hline$\alpha_{1}$ & -------- & -------- & $\begin{array}{l}-0.1254 \\
(-3.371)\end{array}$ & $\begin{array}{l}-0.0315 \\
(-4.384)\end{array}$ \\
\hline$\alpha_{2}$ & -------- & -------- & $\begin{array}{c}-1.155 \\
(-21.184) \\
\end{array}$ & $\begin{array}{l}-0.4169 \\
(-10.18)\end{array}$ \\
\hline$\omega_{0}$ & $\begin{array}{l}0.0047 \\
(3.347)\end{array}$ & $\begin{array}{l}0.00003 \\
(6.418)\end{array}$ & $\begin{array}{l}0.0032 \\
(1.524)\end{array}$ & $\begin{array}{c}0.00004 \\
(6.108)\end{array}$ \\
\hline$\omega_{1}$ & $\begin{array}{l}2.2086 \\
(2.623) \\
\end{array}$ & $\begin{array}{c}0.7708 \\
(5.4333) \\
\end{array}$ & $\begin{array}{l}3.9393 \\
(1.283)\end{array}$ & $\begin{array}{c}0.7475 \\
(6.1468) \\
\end{array}$ \\
\hline$\gamma$ & -------- & $\begin{array}{l}-0.3222 \\
(-6.714)\end{array}$ & -------- & $\begin{array}{l}-0.2896 \\
(-5.675)\end{array}$ \\
\hline$\psi$ & -------- & $\begin{array}{c}0.1916 \\
(14.100)\end{array}$ & -------- & $\begin{array}{c}0.1897 \\
(13.413)\end{array}$ \\
\hline$\lambda$ & -------- & $\begin{array}{c}0.2176 \\
(11.483)\end{array}$ & -------- & $\begin{array}{c}0.2191 \\
(12.068)\end{array}$ \\
\hline $\begin{array}{l}\text { Log-Likelihood } \\
\text { Function }\end{array}$ & 1127.51 & 2128.99 & 1185.47 & 2150.58 \\
\hline $\mathrm{SIC}$ & 1116.75 & 2107.48 & 1171.12 & 2125.48 \\
\hline
\end{tabular}

Estimation is carried out using maximum-likelihood. Heteroskedasticity-Consistent t-statistics are in parentheses. 
Third, Table 4 also analyses the effect on changing the specification of $\theta_{t}$. There is no much difference in the parameters when we compare either model $i$ against model $i i$ (single mean reversion) or model iii against model iv (double mean reversion). The SIC statistic reaches a slightly higher value for both situations when $\theta_{t}$ is not constant. It also holds that the parameter $\gamma$ is negative and significant. This result leads to a mean reversion to the MRO rate in terms of the mean of the jump size.

Fourth, if we analyse the ARCH effect and take, for instance, the value of $\omega_{1}$ equals 0.648 from model $i v$ in Table 4 then it reflects both a high and persistent timevarying volatility defined through the ARCH (1) structure. Note that this value is similar across all models except for the ARCH-Gaussian ones - models 1 and 3 from Table 3 that we will study later. With regard to models 2 and 4 in Table 3, their respective values of $\omega_{1}, 0.771$ and 0.747 respectively, are higher than in Table 4 . It means that going from a constant to a time-varying intensity (a piecewise function in this case) parameter drives to a decrease in the ARCH persistence. Under model $i v$, it holds that the average of the daily $\mathrm{ARCH}$ variance series is about 0.023 which is similar to 0.020 corresponding to the sample variance of $\Delta r_{t}$ in Table $1\left(0.142^{2}\right)$. If we compute the mean of this daily conditional variance series at different periods measured according to the dummy variables in equation (9), it is verified that the daily variance scores the highest value at the end of the maintenance period each month. We obtain a daily variance mean of 0.042 for the maintenance period or $D_{t}^{m p}=1$. Meanwhile, the daily variance averages of $0.026,0.023$ and 0.015 would be associated with $D_{t}^{m}=1, D_{t}^{\text {meet }}=1$ and $D_{t}^{o}=1$ respectively.

Fifth, a comparison of the ARCH-Gaussian - models 1 and 3 in Table 3 - and any ARCH-Poisson-Gaussian model reveals a sharp drop in the conditional variance once jumps are implemented. Note that the ARCH (1) process drives to a large value of $\omega_{1}$ and higher than 1 under models 1 and 3. This fact implies that jumps account for a substantial component of the volatility as the equation (6) confirms.

Sixth, we can also be interested in knowing how the jump volatility $\sigma_{t, J}^{2}$ in equation (5) does change due to changes in the jump intensity. We would expect the derivative 
Table 4 ARCH-Poisson-Gaussian process with $\lambda_{t}$ as a piecewise function

Daily data of EONIA rate covering the period January 1999 to December 2003. This table contains the ARCHPoisson-Gaussian process under different features of both $\mu_{t}$ and $\theta_{t}$. Our more general ARCH-Poisson-Gaussian process is specified as follows:

$$
\Delta r_{t}=\mu_{t}+\sigma_{t} z_{t}+J_{t} \Delta n_{t} ; \quad z_{t} \sim N(0,1) ; J_{t} \sim N\left(\theta_{t}, \psi^{2}\right) ; z_{t} \text { and } J_{t} \text { are independent }
$$

where $\sigma_{t}^{2}=\omega_{0}+\omega_{1}\left[\Delta r_{t-1}-E_{t-2}\left(\Delta r_{t-1}\right)\right]^{2}$ and both $\mu_{t}$ and $\theta_{t}$ are defined in the table below.

\begin{tabular}{|c|c|c|c|c|}
\hline & \multicolumn{2}{|c|}{$\mu_{t}=\alpha\left(r_{t-1}-r_{t-1}^{*}\right)$} & \multicolumn{2}{|c|}{$\mu_{t}=\alpha_{1}\left(r_{t-1}-r_{t-1}^{*}\right)^{+}+\alpha_{2}\left(r_{t-1}-r_{t-1}^{*}\right)^{-}$} \\
\hline Parameters & $\begin{array}{l}\text { Model } i \\
\theta_{t}=\theta\end{array}$ & $\begin{array}{c}\text { Model } i i \\
\theta_{t}=\gamma\left(r_{t-1}-r_{t-1}^{*}\right)\end{array}$ & $\begin{array}{c}\text { Model iii } \\
\qquad \theta_{t}=\theta\end{array}$ & $\begin{array}{c}\text { Model } i v \\
\theta_{t}=\gamma\left(r_{t-1}-r_{t-1}^{*}\right)\end{array}$ \\
\hline$\alpha$ & $\begin{array}{c}-0.046 \\
(-6.601) \\
\end{array}$ & $\begin{array}{l}-0.0344 \\
(-6.365)\end{array}$ & ------- & -------- \\
\hline$\alpha_{1}$ & -------- & -------- & $\begin{array}{l}-0.0465 \\
(-6.244)\end{array}$ & $\begin{array}{l}-0.0388 \\
(-7.832)\end{array}$ \\
\hline$\alpha_{2}$ & ------- & -------- & $\begin{array}{l}-1.0928 \\
(-263.81)\end{array}$ & $\begin{array}{l}-0.9943 \\
(-61.837)\end{array}$ \\
\hline$\omega_{0}$ & $\begin{array}{c}0.00003 \\
(5.123)\end{array}$ & $\begin{array}{l}0.00003 \\
(7.189)\end{array}$ & $\begin{array}{c}0.0004 \\
(10.127)\end{array}$ & $\begin{array}{c}0.0004 \\
(12.833)\end{array}$ \\
\hline$\omega_{1}$ & $\begin{array}{l}0.6240 \\
(5.922) \\
\end{array}$ & $\begin{array}{r}0.6092 \\
(5.542) \\
\end{array}$ & $\begin{array}{l}0.6651 \\
(6.338) \\
\end{array}$ & $\begin{array}{l}0.6482 \\
(6.053) \\
\end{array}$ \\
\hline$\theta$ or $\gamma$ & $\begin{array}{l}-0.0087 \\
(-1.196)\end{array}$ & $\begin{array}{l}-0.4152 \\
(-8.179)\end{array}$ & $\begin{array}{c}-0.009 \\
(-1.170)\end{array}$ & $\begin{array}{l}-0.2758 \\
(-4.406)\end{array}$ \\
\hline$\psi$ & $\begin{array}{c}0.2101 \\
(12.939)\end{array}$ & $\begin{array}{c}0.1903 \\
(14.249)\end{array}$ & $\begin{array}{c}0.2003 \\
(12.294)\end{array}$ & $\begin{array}{c}0.1930 \\
(13.035)\end{array}$ \\
\hline$\delta_{0}$ & $\begin{array}{l}0.1209 \\
(7.841)\end{array}$ & $\begin{array}{l}0.1128 \\
(6.917)\end{array}$ & $\begin{array}{l}0.1025 \\
(7.005)\end{array}$ & $\begin{array}{l}0.1023 \\
(7.209)\end{array}$ \\
\hline$\delta_{1}$ & $\begin{array}{c}0.5260 \\
(10.201)\end{array}$ & $\begin{array}{c}0.5271 \\
(11.546)\end{array}$ & $\begin{array}{c}0.5500 \\
(10.714)\end{array}$ & $\begin{array}{c}0.5347 \\
(10.431)\end{array}$ \\
\hline$\delta_{2}$ & $\begin{array}{l}0.5257 \\
(8.838) \\
\end{array}$ & $\begin{array}{l}0.5076 \\
(9.830) \\
\end{array}$ & $\begin{array}{l}0.5233 \\
(8.486) \\
\end{array}$ & $\begin{array}{l}0.5306 \\
(8.460) \\
\end{array}$ \\
\hline$\delta_{3}$ & $\begin{array}{l}0.1421 \\
(3.582) \\
\end{array}$ & $\begin{array}{r}0.1309 \\
(4.239) \\
\end{array}$ & $\begin{array}{r}0.1344 \\
(3.493) \\
\end{array}$ & $\begin{array}{r}0.1476 \\
(3.568) \\
\end{array}$ \\
\hline$\delta_{4}$ & $\begin{array}{c}0.8307 \\
(10.345)\end{array}$ & $\begin{array}{c}0.8060 \\
(10.055)\end{array}$ & $\begin{array}{c}0.8763 \\
(10.372)\end{array}$ & $\begin{array}{c}0.8847 \\
(10.998)\end{array}$ \\
\hline$\delta_{5}$ & $\begin{array}{l}0.3896 \\
(2.741) \\
\end{array}$ & $\begin{array}{r}0.3569 \\
(3.080) \\
\end{array}$ & $\begin{array}{r}0.4368 \\
(2.843) \\
\end{array}$ & $\begin{array}{l}0.4373 \\
(3.367) \\
\end{array}$ \\
\hline $\begin{array}{c}\text { Log-Likelihood } \\
\text { Function }\end{array}$ & 2186.46 & 2222.14 & 2237.55 & 2256.05 \\
\hline SIC & 2147.01 & 2182.69 & 2194.51 & 2218.01 \\
\hline
\end{tabular}

Estimation is carried out using maximum-likelihood. Heteroskedasticity-Consistent t-statistics are in parentheses. The coefficients $\delta_{1}, \delta_{2}, \delta_{3}, \delta_{4}, \delta_{5}$ and $\delta_{0}$ denote respectively the following probabilities of jump: (i) the last four days of the maintenance period, (ii) the last two days of the month, (iii) the day of the meeting of the ECB's Governing Council and next day, (iv) the day of the meeting of the ECB's Governing Council or the following day when they coincide with the last four days of a maintenance period, (v) the day of the meeting of the ECB's Governing Council or the following day when they coincide with the last two days of the month and (vi) the remaining days. In short, the intensity of the jump is defined as follows:

$$
\lambda_{t}=\delta_{0} D_{t}^{0}+\delta_{1} D_{t}^{m p}+\delta_{2} D_{t}^{m}+\delta_{3} D_{t}^{\text {meet }}+\delta_{4} D_{t}^{\text {meet }+m p}+\delta_{5} D_{t}^{\text {meet }+m}
$$

where $D_{t}^{0}, D_{t}^{m p}, D_{t}^{m}, D_{t}^{\text {meet }}, D_{t}^{\text {meet }+m p}, D_{t}^{\text {meet }+m}$ are dummy variables. 
$\partial \sigma_{t, J}^{2} / \partial \lambda_{t}$ to be positive. It holds that $\partial \sigma_{t, J}^{2} / \partial \lambda_{t}>0 \Leftrightarrow \lambda_{t}<\frac{1}{2}\left(1+\psi^{2} / \theta_{t}^{2}\right)$. Consider, for instance, the situation of $\theta_{t}=\theta$ and $\lambda_{t}$ to be time varying - models $i$ and iii from Table 4 , then it is shown that $\lambda_{t}$ verifies the above condition.

Last but not least, there are several reasons why jumps are more probable to occur on certain days than on others. Henceforth, our comments will be related to model $i v$ in Table 4. We examined the probability of the occurrence of a jump during the final days of the maintenance period $\left(\delta_{1}\right)$, on the last days of each month $\left(\delta_{2}\right)$, on the day the Governing Council of the ECB meets and the following day $\left(\delta_{3}\right)$ and the remaining days $\left(\delta_{0}\right)$. As one might expect, the jump arrival intensity is very high at the end of the maintenance period, $\delta_{1}=0.535$. Likewise, the parameter which measures the jump intensity at the end of the month, $\delta_{2}$, is nearly just as high as $\delta_{1}$, having grouped all the calendar effects (end of month, quarter, half-year and year) into a single effect (end of month effect). The lowest jump intensity is on the day of the meeting and the following day, $\delta_{3}=0.148$. This result must be taken with caution because this parameter covers all the meetings, both those after which there has been a change in the official rate and those after which no change has taken place. The results of the parameters $\delta_{4}$ and $\delta_{5}$, which indicate the jump intensity when the end of the maintenance period and the end of the month coincide with a meeting respectively, are not important results due to the low percentage that they represent of the whole. Specifically, $17 \%$ of ends of month coincide with a meeting, while only $8 \%$ of meetings coincide with an end of month. Moreover, $28 \%$ of the ends of maintenance periods match up with a meeting, and vice versa. The introduction of the dummy variables $D_{t}^{\text {meet+mp }}$ and $D_{t}^{\text {meet+m }}$ is due to the fact that these dummies let us to withdraw coincidence with other effects from each effect ${ }^{8}$. The coefficient $\delta_{0}$ measures the impact by the occurrence of jumps due to those possible effects that could happen on the remaining days. It gives a value of 0.102 , that is, the lowest possibility of a jump occurs on those days in which none of the analysed effects takes place.

\footnotetext{
${ }^{8}$ The total of days corresponding to each group are: 172 data for $D_{t}^{m p}=1,100$ observations for $D_{t}^{m}=1,152$ for $D_{t}^{\text {meet }}=1,789$ for $D_{t}^{o}=1,68$ for $D_{t}^{\text {meet }+m p}=1$ and finally, 20 for $D_{t}^{\text {meet }+m}=1$.
} 
Finally, another important question is: does the double mean reversion specification for the conditional mean in equation (8) help to capture the nonlinearity behavior of the drift for $\Delta r_{t}$ ?. We compute the daily log-likelihood ratio $\left(L R_{t}\right)$ statistic between the following two models: the restricted (single mean reversion) and unrestricted (double mean reversion) specifications with $\theta_{t}=\theta$ corresponding to the models $i$ and iii in Table 4 respectively. Note that the restricted version is the case of $\alpha_{1}=\alpha_{2}$ in equation (8). So, $L R_{t}$ is defined as twice the difference between the daily log-likelihood values of the two models. Note that when $L R_{t}>0$ the double mean reversion process performs better than only a mean reversion process. Summation of these daily likelihood ratios over the whole sample generates the likelihood ratio test (LRT) statistic for the null hypothesis of one mean reversion against the alternative of double mean reversion. Here, the statistic $L R T \sim \chi^{2}(1)$ with value equals 102.18 gives a p-value of zero, so the null hypothesis of one mean reversion is rejected. Figure 2 plots the daily $L R_{t}$ series where we can see that there are more positive $-61 \%$ over the whole sample - than negative values. Note that the highest values in absolute value of $L R_{t}$ are reached when $L R_{t}>0$. A deeper analysis drives to split the series in Figure 2 into 4 groups: (a) the period containing the end of the month or those days verifying that $D_{t}^{m}=1$; (b) the period for the meetings where in this case we join those days corresponding to any of the following groups: $D_{t}^{\text {meet }}=1$, $D_{t}^{m e e t+m p}=1$ or $D_{t}^{\text {meet+m }}=1$; (c) those days for the maintenance period or $D_{t}^{m p}=1$ and finally; (d) those days satisfying that $D_{t}^{o}=1$. It holds that the number of positive values is higher in every group except for the maintenance period. Summing up, the percentage of each group's positive values are respectively: $69 \%, 51.67 \%{ }^{9}, 40.12 \%$ and $67.55 \%$.

\subsection{Overreaction}

Modifying the specification for $\lambda_{t}$ in (9) we can easily test if the EONIA rate reacts strongly to large moves, that is, whether there is a quick reaction in the opposite direction.

\footnotetext{
${ }^{9}$ If we consider for the second group, or meeting period, only the case of $D_{t}^{\text {meet }}=1$ then the percentage of positive values is $54 \%$ that is slightly higher.
} 


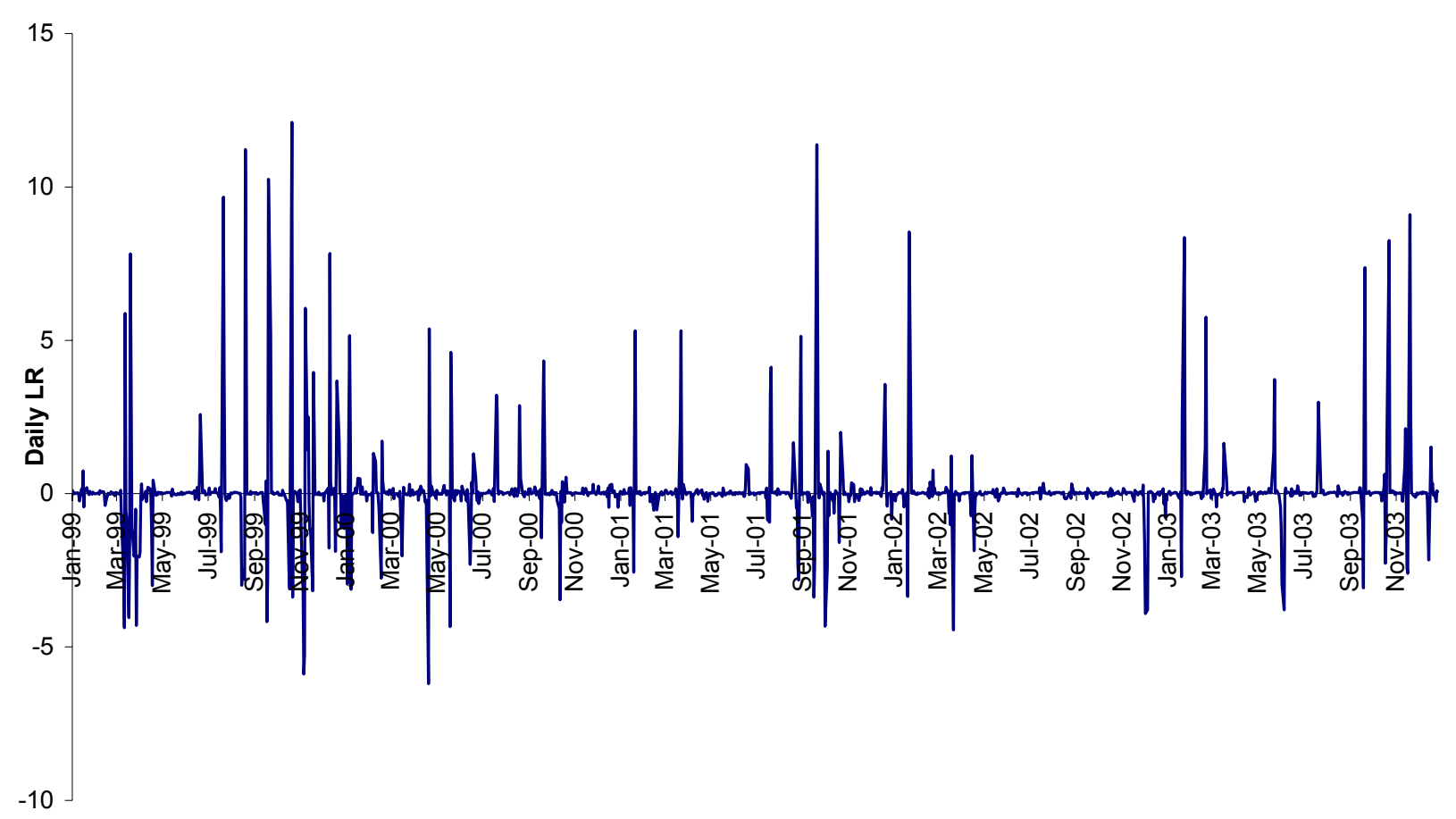

This phenomenon is known as overreaction. The existence of overreaction would mean that the direction of the interest rate would be predictable after large moves. If there is overreaction, the probability of a jump in the opposite direction to the previous large movement in the interest rate would weight more than the probability of a jump in the same direction as the previous movement. To verify this, we modify the jump intensity in the same way as Das (2002) by making the intensity parameter as a function of the product of the current and previous change in the interest rate, thus:

$$
\lambda_{t}=\delta_{0}+\delta_{1} R_{t}^{+}+\delta_{2} R_{t}^{-}
$$

where

$$
R_{t}^{+}=\max \left[0,\left(r_{t}-r_{t-1}\right)\left(r_{t-1}-r_{t-2}\right)\right] \text { and } R_{t}^{-}=\min \left[0,\left(r_{t}-r_{t-1}\right)\left(r_{t-1}-r_{t-2}\right)\right]
$$

where $\delta_{1}$ and $\delta_{2}$ denote respectively the magnitude of the "continuation" and the "reverse" in the direction of the changes. Table 5 shows that the value of the parameters $\delta_{1}$ and $\delta_{2}$ are both significant, indicating that they have a strong impact on the jump 
intensity. The value of $\delta_{1}$ shows that the "level of continuation" in the changes equals 0.382 , while the "level of overreaction" - measured through $\delta_{2}$ - reaches a value of 0.606 , implying that the overreaction effect is twice as big as the "continuation" effect. This leads us to the conclusion that the EONIA rate overreacts to large moves and hence, the rejection of the martingale hypothesis.

\section{Table 5 Overreaction}

The parameters have been estimated using the following econometric specification:

$$
\begin{aligned}
& \Delta r_{t}=\mu_{t}+\sigma_{t} z_{t}+J_{t} \Delta n_{t} ; J_{t} \sim N\left(\theta_{t}, \psi^{2}\right) ; z_{t} \text { and } J_{t} \text { are independent where } \\
& \mu_{t}=\alpha_{1}\left(r_{t-1}-r_{t-1}^{*}\right)^{+}+\alpha_{2}\left(r_{t-1}-r_{t-1}^{*}\right)^{-}, \sigma_{t}^{2}=\omega_{0}+\omega_{1}\left[\Delta r_{t-1}-E_{t-2}\left(\Delta r_{t-1}\right)\right]^{2} \text { and } \theta_{t}=\gamma\left(r_{t-1}-r_{t-1}^{*}\right) .
\end{aligned}
$$

\begin{tabular}{|c|c|c|c|c|c|c|c|c|c|c|c|}
\hline Parameter & $\alpha_{1}$ & $\alpha_{2}$ & $\omega_{0}$ & $\omega_{1}$ & $\gamma$ & $\psi$ & $\delta_{0}$ & $\delta_{1}$ & $\delta_{2}$ & $\begin{array}{c}\text { Log- } \\
\text { Likelihood } \\
\text { Function }\end{array}$ & SIC \\
\hline Value & $\begin{array}{c}-0.0344 \\
(-4.085)\end{array}$ & $\begin{array}{c}-0.0411 \\
(-0.663)\end{array}$ & $\begin{array}{c}0.0004 \\
(12.943)\end{array}$ & $\begin{array}{c}0.1216 \\
(1.656)\end{array}$ & $\begin{array}{c}-0.5783 \\
(-8.129)\end{array}$ & $\begin{array}{c}0.1867 \\
(15.733)\end{array}$ & $\begin{array}{c}0.1262 \\
(8.838)\end{array}$ & $\begin{array}{c}0.3824 \\
(7.166)\end{array}$ & $\begin{array}{c}0.6064 \\
(8.853)\end{array}$ & 2221.41 & 2189.13 \\
\hline
\end{tabular}

Estimation is carried out using maximum-likelihood. Heteroskedasticity-Consistent t-statistics are in parentheses. The variable $\delta_{1}$ is the continuation coefficient and the variable $\delta_{2}$ is the reversal component. The intensity of the jump is defined as $\lambda_{t}=\delta_{0}+\delta_{1} R_{t}^{+}+\delta_{2} R_{t}^{-}$where $R_{t}^{+}=\max \left[0,\left(r_{t}-r_{t-1}\right)\left(r_{t-1}-r_{t-2}\right)\right]$ and $R_{t}^{-}=\min \left[0,\left(r_{t}-r_{t-1}\right)\left(r_{t-1}-r_{t-2}\right)\right]$.

\section{Autoregressive conditional jump intensity}

In section 4 , we have obtained a model for the EONIA rate that incorporates the end of the month effect, the end of the maintenance period effect, and the ECB's meetings in the intensity parameter. Note that the first two effects above can be set as seasonal ones in the sense that they are exhibited each month. In this section, we will generalize the piecewise equation (9) for the dynamics of $\lambda_{t}$ with a truly time varying function. In concrete, we will implement the autoregressive conditional jump intensity (ARJI), see Chan and Maheu (2002) and Maheu and McCurdy (2004). The idea behind our first model or equation (9) was trying to understand the dynamics of the jump intensity and so, which factors may affect its behavior. In this section, we are interested in a more general model of $\lambda_{t}$ in the sense of capturing better the pattern of the EONIA series but also, a model to forecast that will be used later in section 8 , specifically, for the out-of-sample performance 
of the volatility. It is very important to search for a parsimonious structure of $\lambda_{t}$. We can state that those features that could be characterised as a seasonal pattern are both the end of the month and the maintenance period since they affect the changes in the EONIA rate. Nevertheless, the meeting effect does not support this kind of seasonal evidence. The reason is that in our estimation period, the number of times the target rate changed was 15 over a total of 120 meetings for a period of 5 years ( 2 meetings per month). It means that although we know the date of the meeting each month, the frequency of a change in the interest rate is very low in order to consider important the meeting dates by fixing a dummy variable for these dates. In short, it means to leave out $D_{t}^{\text {meet }}$ while keeping both $D_{t}^{m p}$ and $D_{t}^{m}$ for the new specification of $\lambda_{t}$.

To reinforce this decision of exclusion, remember that we have assumed in this paper for the target rate to be an exogenous variable instead of being endogenous. Modeling the target rate is beyond the scope of this paper. If we were interested in that case, we would need additional information such as the evolution of the inflation rate, the GDP rate and so on. The dynamics of these macroeconomic variables would be followed by the ECB for the decision of keeping constant or changing the target rate in each meeting. In short, since we have not established any rule for the ECB about its decision on altering the target rate, this is the reason why we do not incorporate this effect into the jump intensity behavior if we are interested in a model for the EONIA in order to foresee. This idea of forecasting the target rate given the information until nowadays containing the time series of the target rate but also some exogenous variables, such as unemployment, production and so on, can be seen in the study of the daily federal funds (FF) target rate by Hamilton and Jordá (2002). Another more recent study of prediction can be seen in Sarno et al. (2005) for the FF rate where the standard macroeconomic variables, which are useful for the monetary policy decisions, are not included in the information set but it does contain the FF target rate, other US interest rates, FF futures rates and lagged values of the FF rate. It is important to mention that our paper does not aim to forecast the level of the EONIA rate among several candidate models. However, we will show in subsection 6.1 
how to predict future values for $\lambda_{t}$ conditioned to the information set at present which are necessary for the prediction of level of the EONIA rate ${ }^{10}$.

Finally, we will study the behavior of $\lambda_{t}$ around the days of the ECB's meetings. Under the new modeling of $\lambda_{t}$, i.e. ARJI dynamics, we can obtain a daily time varying expost probability of the occurrence of a jump. A higher value of this probability gives a more clear proof of jump. This probability is useful to check the existence of jumps around those meetings where it really happen a change in the target rate.

\subsection{The model}

The dynamics for $\lambda_{t}$ is given by

$$
\lambda_{t}=\delta_{0}+\delta_{1} \lambda_{t-1}+\delta_{2} \xi_{t-1}+\delta_{3} D_{t}^{m p}+\delta_{4} D_{t}^{m}
$$

where $D_{t}^{m p}\left(D_{t}^{m}\right)$ corresponds to a dummy variable for the last four days of the maintenance period (the last two days of each month) ${ }^{11}$. The standard ARJI model is nested in equation (10), just for the case of $\delta_{3}=\delta_{4}=0$. The coefficient $\delta_{1}$ measures the level of persistence over the arrival of jump events (jump clustering). The term $\xi_{t-1}$ is known as the jump intensity innovation and it is defined as

$$
\xi_{t-1} \equiv E_{t-1}\left[\Delta n_{t-1}\right]-\lambda_{t-1}=P\left(\Delta n_{t-1}=1 \mid \Phi_{t-1}\right)-\lambda_{t-1}
$$

where $P\left(\Delta n_{t-1}=1 \mid \Phi_{t-1}\right)$ is called the filter and it is the ex-post inference on $\Delta n_{t-1}$ given the information set $\Phi_{t-1}$, while $\lambda_{t-1}$ is by definition the conditional expectation of $\Delta n_{t-1}$

\footnotetext{
${ }^{10}$ Closed-form formulae to the daily forecasting of the EONIA rate, that is $E_{t}\left[r_{t+k}\right]$ where $k \geq 1$, are also obtained according to the new specification of $\lambda_{t}$ - see equation (10) - though they are not reported here in order to shorten the paper. These expressions are available upon request.

${ }^{11}$ Those days satisfying $D_{t}^{\text {meet }+m p}=1\left(D_{t}^{\text {meet+m }}=1\right)$ in equation (9), now they are grouped into the maintenance period (the end of the month) and so, verifying that $D_{t}^{m p}=1\left(D_{t}^{m}=1\right)$ in (10).
} 
given $\Phi_{t-2}$. This implies that $\xi_{t}$ is a martingale difference sequence with respect to $\Phi_{t-1}$, that is, $E_{t-1}\left[\xi_{t}\right]=0$. It also holds that $E\left[\xi_{t}\right]=0$ and $\operatorname{Cov}\left(\xi_{t}, \xi_{t-i}\right)=0, \forall i$. In short, the parameter $\delta_{2}$ measures the effect of the most recent intensity residual or the change in the conditional forecast of $\Delta n_{t-1}$ given the last available information set $\Phi_{t-1}$ which is larger than $\Phi_{t-2}$.

Equation (10) will be a stationary process if it holds that $\left|\delta_{1}\right|<1$. Denote as $x_{t}$ the vector $\left(D_{t}^{m p}, D_{t}^{m}\right)$. Note that $x_{t}$ has only three possible values: $(0,0),(1,0)$ and $(0,1)$. By the law of iterated expectations, the unconditional probability of one jump $\left(\lambda_{t}\right)$ is equal to

$$
E\left[\lambda_{t}\right]=E\left[E\left[\lambda_{t} \mid x_{t}\right]\right]=\pi_{0,0} E\left[\lambda_{t} \mid(0,0)\right]+\pi_{1,0} E\left[\lambda_{t} \mid(1,0)\right]+\pi_{0,1} E\left[\lambda_{t} \mid(0,1)\right]
$$

where $\pi_{i, j}$ denotes the probability corresponding to the value $(i, j)$ for $x_{t}$ such that $\pi_{0,0}+\pi_{1,0}+\pi_{0,1}=1$. Hence,

$$
E\left[\lambda_{t}\right]=\frac{\delta_{0}+\pi_{1,0} \delta_{3}+\pi_{0,1} \delta_{4}}{1-\delta_{1}}
$$

Let $E_{t}\left[\lambda_{t+k}\right]$ denote the forecasts of the future jump intensities conditioned to nowadays that is denoted as period $t$. Since $E_{t}\left[\lambda_{t+1}\right]=\lambda_{t+1}$, then for the case of $k \geq 2$ we have:

$$
E_{t}\left[\lambda_{t+k}\right]=\delta_{0}\left(\frac{1-\delta_{1}^{k-1}}{1-\delta_{1}}\right)+\delta_{1}^{k-1} \lambda_{t+1}+\delta_{3} \sum_{j=2}^{k} \delta_{1}^{k-j} D_{t+j}^{m p}+\delta_{4} \sum_{j=2}^{k} \delta_{1}^{k-j} D_{t+j}^{m}
$$

Finally, once we have observed $r_{t}$ and using the Bayes rule, we can infer the expost or filtered probability of the occurrence of one jump at time $t$ defined as

$$
P\left(\Delta n_{t}=1 \mid \Phi_{t}\right)=\frac{f\left(\Delta r_{t} \mid \Delta n_{t}=1, \Phi_{t-1}\right) P\left(\Delta n_{t}=1 \mid \Phi_{t-1}\right)}{f\left(\Delta r_{t} \mid \Phi_{t-1}\right)}
$$


where $P\left(\Delta n_{t}=1 \mid \Phi_{t-1}\right)=\lambda_{t}$ and so, $P\left(\Delta n_{t}=0 \mid \Phi_{t}\right)=1-P\left(\Delta n_{t}=1 \mid \Phi_{t}\right)$.

\subsection{Results}

Table 6 reports the parameter estimates for the ARCH-Poisson-Gaussian model, where $\mu_{t}$ is a double mean reverting process and $\theta_{t}$ shows mean reversion, under the dynamics of $\lambda_{t}$ in the equation (10) or model B and the restricted version of (10) given by $\delta_{1}=\delta_{2}=0$ or model A. Note that $\lambda_{t}$ in model A results to be a piecewise function similar but easier than $\lambda_{t}$ of equation (9) - see model $i v$, Table $4-$.The results show evidence of time-variation in the arrival of jump events since all the parameters of $\lambda_{t}$ in model $\mathrm{B}$ are significantly different from zero. We observe that the same conclusions for the analysis of the double mean reversion in Table 4 also maintain here. The parameter $\gamma$ which defines the speed of reversion of $\theta_{t}$ is also negative. Note that the interpretation of the jump intensity for either the maintenance period ( $\mathrm{mp}$ ) or the end of the month (m) from model A is different. These effects measured respectively by the parameters $\delta_{3}$ and $\delta_{4}$ show the increment that $\lambda_{t}$ suffers when any of these effects does occur, that is, $\lambda_{t}=\delta_{0}+\delta_{3}$ for the maintenance period and $\lambda_{t}=\delta_{0}+\delta_{4}$ for the end of the month. We can also appreciate how equation (10), or model B, is better than its restricted version or model A. The corresponding LRT statistic has a chi-square distribution with two degrees of freedom and a value of 48.6 (pvalue equals zero) and hence, model B beats model A.

If we analyse the contribution of each component to the total variance in equation (5), that is, $\sigma_{t, J}^{2} / V_{t-1}\left(\Delta r_{t}\right)$ and $\sigma_{t}^{2} / V_{t-1}\left(\Delta r_{t}\right)$ which represent the proportion of the conditional variance explained by the jump and the $\mathrm{ARCH}$ component respectively. It is verified for model B that the mean (median) of the series $\sigma_{t, J}^{2} / V_{t-1}\left(\Delta r_{t}\right)$ is $0.817(0.976)$ that is much higher than the mean (median) of $\sigma_{t}^{2} / V_{t-1}\left(\Delta r_{t}\right)$ which is $0.161(0.033)$. The standard deviation for both series is nearly the same, a value of 0.288 . 
Table 6 ARCH-Poisson-Gaussian process with $\lambda_{t}$ time varying

Daily data of EONIA rate covering the period January 1999 to December 2003. The ARCH-Poisson-Gaussian process estimated is specified as follows:

$\Delta r_{t}=\mu_{t}+\sigma_{t} z_{t}+J_{t} \Delta n_{t} ; \quad z_{t} \sim N(0,1) ; \quad J_{t} \sim N\left(\theta_{t}, \psi^{2}\right)$

where $z_{t}$ and $J_{t}$ are independent; $\mu_{t}=\alpha_{1}\left(r_{t-1}-r_{t-1}^{*}\right)^{+}+\alpha_{2}\left(r_{t-1}-r_{t-1}^{*}\right)^{-}$; $\sigma_{t}^{2}=\omega_{0}+\omega_{1}\left[\Delta r_{t-1}-E_{t-2}\left(\Delta r_{t-1}\right)\right]^{2}, \quad \theta_{t}=\gamma\left(r_{t-1}-r_{t-1}^{*}\right) \quad$ and $\lambda_{t}$ is different in each model: $\lambda_{t}=\delta_{0}+\delta_{3} D_{t}^{m p}+\delta_{4} D_{t}^{m} \quad$ (model A) and $\lambda_{t}=\delta_{0}+\delta_{1} \lambda_{t-1}+\delta_{2} \xi_{t-1}+\delta_{3} D_{t}^{m p}+\delta_{4} D_{t}^{m}(\operatorname{model~B})$

\begin{tabular}{|c|c|c|}
\hline Parameter & Model A & Model B \\
\hline$\alpha_{1}$ & $\begin{array}{l}-0.0379 \\
(-7.486)\end{array}$ & $\begin{array}{l}-0.0383 \\
(-7.330)\end{array}$ \\
\hline$\alpha_{2}$ & $\begin{array}{c}-1.0574 \\
(-87.213)\end{array}$ & $\begin{array}{c}-0.9095 \\
(-44.226) \\
\end{array}$ \\
\hline$\omega_{0}$ & $\begin{array}{l}0.00004 \\
(6.685)\end{array}$ & $\begin{array}{l}0.00003 \\
(7.763)\end{array}$ \\
\hline$\omega_{1}$ & $\begin{array}{l}0.7313 \\
(6.035)\end{array}$ & $\begin{array}{l}0.7011 \\
(7.166)\end{array}$ \\
\hline$\gamma$ & $\begin{array}{l}-0.3218 \\
(-2.812)\end{array}$ & $\begin{array}{l}-0.4878 \\
(-13.057)\end{array}$ \\
\hline$\psi$ & $\begin{array}{c}0.1944 \\
(12.478)\end{array}$ & $\begin{array}{c}0.1920 \\
(11.621)\end{array}$ \\
\hline$\delta_{0}$ & $\begin{array}{l}0.1038 \\
(7.180) \\
\end{array}$ & $\begin{array}{l}0.0317 \\
(4.249) \\
\end{array}$ \\
\hline$\delta_{1}$ & ------ & $\begin{array}{l}0.4031 \\
(7.373)\end{array}$ \\
\hline$\delta_{2}$ & ------ & $\begin{array}{l}0.6140 \\
(7.090)\end{array}$ \\
\hline$\delta_{3}$ & $\begin{array}{c}0.4965 \\
(10.080)\end{array}$ & $\begin{array}{l}0.2806 \\
(8.818)\end{array}$ \\
\hline$\delta_{4}$ & $\begin{array}{l}0.3951 \\
(6.774)\end{array}$ & $\begin{array}{l}0.3064 \\
(8.745)\end{array}$ \\
\hline $\begin{array}{l}\text { Log-Likelihood } \\
\text { Function }\end{array}$ & 2248.07 & 2287.39 \\
\hline SIC & 2215.79 & 2247.94 \\
\hline
\end{tabular}

Estimation is carried out using maximum-likelihood. HeteroskedasticityConsistent t-statistics are in parentheses.

Table 7 exhibits the descriptive statistics for both the ex-ante and ex-post probabilities for model B. Ex-post (ex-ante) probabilities higher than 0.5 provide a significant evidence that a jump has occurred (forecast the occurrence of a jump). It is verified for both cases that the total of probabilities higher than 0.5 represent a size about $20 \%$. Looking at the quantiles, the probability of getting a value higher than 0.9 ranges 
from 0.10 to 0.20 for the ex-post probability while it is lower than 0.1 for $\lambda_{t}$. If we regress the ex-post probability onto a constant and $\lambda_{t}$, the slope is significantly different from zero and value of 1.01. The R-squared statistic of this regression is 0.40 . Finally, if we study more carefully those situations where the ex-post probabilities reach values higher than 0.5 - in concrete, an amount of 261 over 1,299 observations in all -, it holds a percentage of $50 \%$ for the maintenance period, $21 \%$ for the end of the month, $27 \%$ for the fifteen meetings over the total where the ECB did really change the official rate - we select here a window of \pm 4 days around the meeting date in order to measure the market reactions and $19 \%$ corresponding to the remaining days.

Table 7 Descriptive statistics of the ex-ante and ex-post probabilities

Daily data of EONIA rate from January 1999 to December 2003.

Ex-ante probability:

$$
\begin{aligned}
& \lambda_{t}=\delta_{0}+\delta_{1} \lambda_{t-1}+\delta_{2} \xi_{t-1}+\delta_{3} D_{t}^{m p}+\delta_{4} D_{t}^{m} \\
& \text { where } \lambda_{t}=P\left(\Delta n_{t}=1 \mid \Phi_{t-1}\right)
\end{aligned}
$$

Ex-post probability:

$$
P\left(\Delta n_{t}=1 \mid \Phi_{t}\right)=\frac{\lambda_{t} f\left(\Delta r_{t} \mid \Delta n_{t}=1, \Phi_{t-1}\right)}{f\left(\Delta r_{t} \mid \Phi_{t-1}\right)}
$$

\begin{tabular}{|l||c||c||}
\hline \multicolumn{1}{|c|}{ Statistics } & $\begin{array}{c}\text { Ex-ante } \\
\text { probability }\end{array}$ & $\begin{array}{c}\text { Ex-post } \\
\text { probability }\end{array}$ \\
\hline Mean & 0.2605 & 0.2454 \\
Median & 0.1636 & 0.0438 \\
Maximum & 0.8888 & 1.0000 \\
Sinimum & 0.0449 & 0.0016 \\
Quantile (75\%) & 0.2178 & 0.3458 \\
Quantile (80\%) & 0.3855 & 0.3743 \\
Quantile (90\%) & 0.5082 & 0.5056 \\
Jarque-Bera & 0.6018 & 0.9958 \\
(p-value) & 161.80 & 372.34 \\
\hline
\end{tabular}




\subsection{Behavior of the probability of jump around the days of the ECB's meetings}

This subsection shows how the intervention held by the ECB's meetings can be a source of jumps. The empirical evidence of jumps can be supported by a high value of the ex-post probability. Table 8 reports these probabilities around the days where an intervention of the official rate occurred for a window of \pm 4 days. We study a total of 15 interventions - the official rate dropped (raised) eight (seven) times - given our sample period. Those situations such that prior to the meeting date the EONIA rate raised a high ex-post probability, they might be interpreted as the market anticipation of changing the official rate in the next meeting. Meanwhile, changes of the interest rates after the meeting date could be interpreted as market reactions due to either the forecast of the interest rate level before the meeting was not the right one, henceforth there is a correction once the new MRO rate is known or those cases where there was no anticipation of a possible change of the MRO rate before holding the meeting. An example for the last case might be the reason of an ascending ex-post probability in the following meetings: October 5, 2000 (upward movement) and May 10, 2001 (downward movement). We can also appreciate that this probability is not always upwards the same day as the meeting. There is also evidence of a cluster of jumps around each meeting excepting March 16, 2000 (upward movement). Finally, Table 8 also exhibits for the selected window around the meeting date that other type of event may have occurred in some cases: the end of the month and the last days of the maintenance period denoted as the symbols " *" and "**" respectively. It happens that the ex-post probabilities tend to be very high in these situations. Note that we could separate the meeting effect against any other effect in every case with the exception of the days around the meetings: August 30, 2001 and September 17, 2001 (descending rates in both dates).

We also carried out another study to understand better how the ECB decisions may affect the value of $\lambda_{t}$. We considered all the meetings occurred - a total of 120 meetings where the rate was intervened in only 15 meetings - and selected a 4-day window before and after the meeting date, that is, a sample of 1,076 data. The idea is to analyse if the impact of this ex-ante probability is different depending on the decision taken from each meeting about changing the MRO rate, that is, either an upward or downward movement. We select a dummy variable $D_{t}^{u p}\left(D_{t}^{\text {down }}\right)$ equals one if day $t$ belongs to the window 
Table 8 Changes in the MRO rate and jumps in the EONIA rate

\begin{tabular}{|c|c|c|c|c|c|c|c|}
\hline \multicolumn{4}{|c|}{ DOWN } & \multicolumn{4}{|c|}{ UP } \\
\hline Date & Ex-post & Date & Ex-post & Date & Ex-post & Date & Ex-post \\
\hline April 2, 1999 & 0.172 & Nov. 2, 2001 & 0.050 & Oct. 29,1999 & $1.000^{*}$ & Jun. 2, 2000 & 0.015 \\
\hline April 5, 1999 & 0.029 & Nov. 5, 2001 & 0.063 & Nov. 1,1999 & 0.251 & Jun. 5, 2000 & 0.012 \\
\hline April 6, 1999 & 0.014 & Nov. 6, 2001 & 1.000 & Nov. 2,1999 & 0.386 & Jun. 6, 2000 & 0.009 \\
\hline April 7, 1999 & 0.999 & Nov. 7, 2001 & 0.339 & Nov. 3, 1999 & 0.053 & Jun. 7, 2000 & 1.000 \\
\hline April 8, 1999 & 0.338 & Nov. 8, 2001 & 0.828 & Nov. 4, 1999 & 0.040 & Jun. 8, 2000 & 0.847 \\
\hline April 9, 1999 & 1.000 & Nov. 9, 2001 & 0.266 & Nov. 5, 1999 & 0.037 & Jun. 9. 2000 & 0.152 \\
\hline April 12, 1999 & 0.369 & Nov. 12, 2001 & 0.032 & Nov. 8, 1999 & 0.045 & Jun. 12, 2000 & 0.132 \\
\hline April 13, 1999 & 0.057 & Nov. 13, 2001 & 0.897 & Nov. 9, 1999 & 0.032 & Jun. 13, 2000 & 0.029 \\
\hline April 14, 1999 & 1.000 & Nov. 14, 2001 & 0.995 & Nov. 10,1999 & 0.031 & Jun. 14, 2000 & 1.000 \\
\hline May 4, 2001 & 0.010 & Nov. 29, 2002 & $1.000^{*}$ & Jan. 28, 2000 & $1.000^{*}$ & Aug. 25, 2000 & 0.122 \\
\hline May 7, 2001 & 0.003 & Dec. 2, 2002 & 0.186 & Jan. 31, 2000 & $0.612^{*}$ & Aug. 28, 2000 & 0.049 \\
\hline May 8, 2001 & 0.003 & Dec. 3, 2002 & 0.932 & Feb. 1, 2000 & 0.154 & Aug. 29, 2000 & 0.012 \\
\hline May 9, 2001 & 0.005 & Dec. 4, 2002 & 1.000 & Feb. 2, 2000 & 0.634 & Aug. 30, 2000 & 0.056 \\
\hline May 10, 2001 & 1.000 & Dec. 5, 2002 & 0.311 & Feb. 3, 2000 & 0.054 & Aug. 31, 2000 & 0.153 \\
\hline May 11, 2001 & 0.999 & Dec. 6, 2002 & 0.130 & Feb. 4, 2000 & 0.267 & Sept. 1, 2000 & 0.229 \\
\hline May 14, 2001 & 0.259 & Dec. 9, 2002 & 0.096 & Feb. 7, 2000 & 0.039 & Sept. 4, 2000 & 0.012 \\
\hline May 15, 2001 & 0.010 & Dec. 10, 2002 & 0.003 & Feb. 8, 2000 & 0.004 & Sept. 5, 2000 & 0.036 \\
\hline May 16, 2001 & 0.097 & Dec. 11, 2002 & 0.004 & Feb. 9, 2000 & 0.008 & Sept. 6, 2000 & 0.011 \\
\hline Aug. 24, 2001 & 0.046 & Feb. 28, 2003 & $0.999^{*}$ & Mar. 10, 2000 & 0.134 & Sept. 29, 2000 & $1.000^{*}$ \\
\hline Aug. 27, 2001 & 0.087 & Mar. 3, 2003 & 0.769 & Mar.13, 2000 & 0.048 & Oct. 2, 2000 & 0.406 \\
\hline Aug. 28, 2001 & 0.015 & Mar. 4, 2003 & 0.587 & Mar. 14, 2000 & 0.018 & Oct. 3, 2000 & 0.090 \\
\hline Aug. 29, 2001 & 1.000 & Mar. 5, 2003 & 0.212 & Mar. 15, 2000 & 0.012 & Oct. 4, 2000 & 0.012 \\
\hline Aug. 30, 2001 & $0.903^{*}$ & Mar. 6, 2003 & 0.099 & Mar. 16, 2000 & 0.003 & Oct. 5, 2000 & 0.952 \\
\hline Aug. 31, 2001 & $0.576^{*}$ & Mar. 7, 2003 & 0.157 & Mar. 17, 2000 & 0.004 & Oct. 6, 2000 & 0.796 \\
\hline Sept. 3, 2001 & 0.235 & Mar. 10, 2003 & 0.018 & Mar. 20, 2000 & 0.081 & Oct. 9, 2000 & 0.183 \\
\hline Sept. 4, 2001 & 0.043 & Mar. 11, 2003 & 1.000 & Mar. 21, 2000 & 0.091 & Oct. 10, 2000 & 0.015 \\
\hline Sept. 5, 2001 & 0.008 & Mar. 12, 2003 & 0.602 & Mar. 22, 2000 & $1.000^{* *}$ & Oct. 11, 2000 & 0.005 \\
\hline Sept. 11, 2001 & 0.009 & May. 30, 2003 & $0.546^{*}$ & April 21, 2000 & $0.605^{\star \star}$ & & \\
\hline Sept. 12, 2001 & 1.000 & Jun. 2, 2003 & 0.097 & April 24, 2000 & 0.215 & & \\
\hline Sept. 13, 2001 & 0.601 & Jun. 3, 2003 & 0.063 & April 25, 2000 & 0.537 & & \\
\hline Sept. 14, 2001 & 0.140 & Jun. 4, 2003 & 1.000 & April 26, 2000 & 0.125 & & \\
\hline Sept. 17, 2001 & 0.037 & Jun. 5, 2003 & 0.528 & April 27, 2000 & 0.263 & & \\
\hline Sept. 18, 2001 & $1.000^{* *}$ & Jun. 6, 2003 & 0.178 & April 28, 2000 & $1.000^{*}$ & & \\
\hline Sept. 19, 2001 & $0.683^{* *}$ & Jun. 9, 2003 & 0.121 & May 1,2000 & 0.263 & & \\
\hline Sept. 20, 2001 & $0.947^{* *}$ & Jun. 10, 2003 & 0.005 & May 2, 2000 & 0.048 & & \\
\hline Sept. 21, 2001 & $0.470^{* *}$ & Jun. 11, 2003 & 0.038 & May 3,2000 & 0.035 & & \\
\hline
\end{tabular}

$P\left(\Delta n_{t}=1 \mid \Phi_{t}\right)$ is the ex-post probability of the occurrence of one jump at time $t$. The meeting days are recorded here in bold. DOWN (UP) contains those dates where the official (MRO) rate has moved downwards (upwards) by intervention. Those probabilities higher than 0.5 around the intervention date ( \pm 4 days) which do not match with either the maintenance or end of the month period have also been recorded in bold. The symbol $(*)$ denotes a date corresponding to any of the last two days of the month while $(* *)$ shows any of the last four days from the maintenance period. 
around a meeting with the decision of ascending (descending) the rate, or zero otherwise. For the decision of neutral stance, we set the dummy variable denoted as $D_{t}^{\text {neutral }}$ having a similar construction as the others. Regression I in Table 9 reports this kind of analysis where the dependent variable is the estimate of $\lambda_{t}$, denoted as $\hat{\lambda}_{t}$, which is obtained from model B in Table 6 . The explanatory variables are: the three dummy variables mentioned above and also, $D_{t}^{m p}$ and $D_{t}^{m}$ if either the end of maintenance or the end of the month period can take place for the selected window (Table 8 exhibits a few situations of coincidence). As a result, the ex-ante probability scores the highest value for downward movements while it seems to be similar for either an upward movement or no intervention in the MRO rate. The adjusted R-squared statistic for this regression is 0.665 . Meanwhile, regression II tries to eliminate the effect of either the maintenance period or the end of the month by adjusting the value of the ex-ante probability. Again, it holds the same conclusion as before. Finally, regression III is the same as regression I but now the dependent variable is the ex-ante probability. Comparing the parameter estimates in both regressions I and III, we can appreciate the largest change of value in the downward intervention where $\beta_{3}$ moves from 0.224 to 0.353 . Surprisingly, this asymmetric behavior about the impact of the official rate reduction by the ECB having more influence on the dynamics of the EONIA rate is also verified in other markets such as the LIBOR rate, see Guan et al. (2005). In this case, the Federal Open Market Committee (FOMC) contributes more in changing the parameters implied in a jump-diffusion specification for the LIBOR rate upon decisions on federal funds rate reductions than other situations.

\section{A regime-switching process with jumps}

So far, a single-regime model is set for the mean-reversion of EONIA to the target rate. This leads to assuming that the speed of reversion, i.e. either $\alpha$ for the single reversion or both $\alpha_{1}$ and $\alpha_{2}$ for the double reversion - see Table 4 for the two cases -, are the same throughout the sample. A more general model such as a regime switching (RS) process has the advantage, for instance, of being more flexible by allowing a different speed of reversion at different times throughout the sample. The RS process lets to capture several patterns which could be hidden under a single regime and hence, a better 
Table 9 The behavior of the time-varying $\lambda_{t}$ around the ECB's meetings

Regression I: $\hat{\lambda}_{t}=\beta_{1} D_{t}^{\text {neutral }}+\beta_{2} D_{t}^{u p}+\beta_{3} D_{t}^{\text {down }}+\beta_{4} D_{t}^{m p}+\beta_{5} D_{t}^{m}+\varepsilon_{t}$; where $\hat{\lambda}_{t}$ is the estimate of the time-varying intensity $\lambda_{t}$ defined in (10) where the coefficients of $\lambda_{t}$ are exhibited in model B from Table 6 .

Regression II: $\lambda_{t}^{*}=\beta_{1} D_{t}^{\text {neutral }}+\beta_{2} D_{t}^{u p}+\beta_{3} D_{t}^{\text {down }}+\varepsilon_{t}$; where $\lambda_{t}^{*}$ is the adjusted $\hat{\lambda}_{t}$ defined as: $\lambda_{t}^{*}=\hat{\lambda}_{t}-\left(\hat{\delta}_{3} D_{t}^{m p}+\hat{\delta}_{4} D_{t}^{m}\right)$ such that $\hat{\delta}_{3}$ and $\hat{\delta}_{4}$ denote the estimates of $\delta_{3}$ and $\delta_{4}$ respectively that correspond to model B in Table 6.

Regression III: similar to regression I but now the dependent variable is the ex-post probability.

\begin{tabular}{|c|c|c|c|c|c|c|}
\hline & $\begin{array}{l}\text { Neutral } \\
\text { meetings }\end{array}$ & $\begin{array}{c}\text { Upward } \\
\text { interventions }\end{array}$ & $\begin{array}{l}\text { Downward } \\
\text { interventions }\end{array}$ & $\begin{array}{c}\text { End of the } \\
\text { maintenance } \\
\text { period }\end{array}$ & $\begin{array}{l}\text { End of the } \\
\text { month }\end{array}$ & \\
\hline & $\beta_{1}$ & $\beta_{2}$ & $\beta_{3}$ & $\beta_{4}$ & $\beta_{5}$ & R-squared \\
\hline Regression I & $\begin{array}{c}0.1509 \\
(32.388)\end{array}$ & $\begin{array}{c}0.1657 \\
(10.657)\end{array}$ & $\begin{array}{c}0.2245 \\
(11.940)\end{array}$ & $\begin{array}{c}0.4178 \\
(40.178)\end{array}$ & $\begin{array}{c}0.3640 \\
(27.335)\end{array}$ & 0.6658 \\
\hline Regression II & $\begin{array}{c}0.1841 \\
(41.465)\end{array}$ & $\begin{array}{c}0.1817 \\
(11.761)\end{array}$ & $\begin{array}{c}0.2361 \\
(12.457)\end{array}$ & ------- & ------ & 0.0089 \\
\hline Regression III & $\begin{array}{c}0.1186 \\
(13.298)\end{array}$ & $\begin{array}{l}0.1825 \\
(4.705)\end{array}$ & $\begin{array}{l}0.3528 \\
(7.996)\end{array}$ & $\begin{array}{c}0.4522 \\
(17.053)\end{array}$ & $\begin{array}{l}0.3950 \\
(8.900)\end{array}$ & 0.3178 \\
\hline
\end{tabular}

$D_{t}$ is a dummy variable: $D_{t}^{\text {neural }}=1, D_{t}^{u p}=1$ and $D_{t}^{\text {down }}=1$ if the four days around the ECB's meetings in which interest rate did not change, increased and decreased respectively. $D_{t}^{m p}=1$ if the last four days of the maintenance period and $D_{t}^{m}=1$ if the last two days of each month. Estimation is carried out using the least squares method. White heteroskedasticity-consistent t-statistics are in parentheses.

understanding of the EONIA dynamics. Empirical evidence reported among others by Gray (1996), Dahlquist and Gray (2000), Ang and Bekaert (2002a, 2002b) find two states characterised by a low-volatility/random-walk (or near random walk) regime where the short rate spends the majority of the time and a high-volatility/mean-reverting regime. A possible answer to this behavior could be found in Mankiw and Miron (1986). They argue that the smoothing actions of the US Fed make the short rate behave like a random walk corresponding to 'normal' periods, that is why the volatility is low under this regime. Nevertheless, when there are extraordinary shocks, interest rates shift to another state or second regime where its volatility is higher and they show a strong mean-reversion.

We develop an extension of our jump process to accommodate regime changes in the dynamics of EONIA. This approach can be found in Das (2002) though our model is 
more general in the sense that we incorporate the ARCH (1) structure, time varying transition probabilities and so forth. In short, the new model accommodates either a single or double mean reversion, jump structure and ARCH (1) within each regime. Gray (1996) implements a generalized RS model with a GARCH $(1,1)$ structure augmented with the level of the interest rate opposite to a low-order ARCH process as in either Cai (1994) or Hamilton and Susmel (1994). Our model will be in the line of these last two works in the sense of implementing an $\mathrm{ARCH}$ (1) structure and keeping the parameter of the ARCH effect, denoted as $\omega_{1}$, to be the same in both states.

\subsection{The model}

Since we postulate a two regime process, let $S_{t}$ denote the unobserved state or latent variable of the system where $S_{t}=0$ or $1 . S_{t}$ is assumed to follow a first-order Markov process with the transition probabilities:

$$
\begin{aligned}
& P\left[S_{t}=0 \mid S_{t-1}=0\right]=p_{00} ; \quad P\left[S_{t}=1 \mid S_{t-1}=0\right]=1-p_{00} \\
& P\left[S_{t}=1 \mid S_{t-1}=1\right]=p_{11} ; \quad P\left[S_{t}=0 \mid S_{t-1}=1\right]=1-p_{11} .
\end{aligned}
$$

where $p_{00}=\exp \left(q_{0}\right) /\left(1+\exp \left(q_{0}\right)\right)$ and $p_{11}=\exp \left(q_{1}\right) /\left(1+\exp \left(q_{1}\right)\right)$ are defined as logistic functions. Note that these transition probabilities are constant at first. We will also implement the RS model with time-varying transition probabilities. This augmented model will be shown in this section later.

For our ARCH-Poisson-Gaussian with shifting regime specification, we will impose several restrictions on the number of the parameters and also easier specifications for the behavior of $\lambda_{t}$ in order to make the estimation of the new model feasible as we will comment later. We will implement for $\mu_{t}$ either a simple mean reversion process, that is 


$$
\begin{aligned}
& \mu_{S_{t}, t}=\alpha^{S_{t}}\left(r_{t-1}-r_{t-1}^{*}\right) ; \\
& \alpha^{S_{t}}=\alpha^{0}\left(1-S_{t}\right)+\alpha^{1} S_{t} ;
\end{aligned}
$$

or a double mean reversion process:

$$
\begin{aligned}
& \mu_{S_{t}, t}=\alpha_{1}^{S_{t}}\left(r_{t-1}-r_{t-1}^{*}\right)^{+}+\alpha_{2}^{S_{t}}\left(r_{t-1}-r_{t-1}^{*}\right)^{-} \\
& \alpha_{k}^{S_{t}}=\alpha_{k}^{0}\left(1-S_{t}\right)+\alpha_{k}^{1} S_{t}
\end{aligned}
$$

where $k=1,2$. For the ARCH (1) process defined in (7), we will only assume a shifting regime specification for the parameter $\omega_{0}$ :

$$
\begin{aligned}
& \sigma_{S_{t}, t}^{2}=\omega_{0}^{S_{t}}+\omega_{1}\left[\Delta r_{t-1}-E_{t-2}\left(\Delta r_{t-1}\right)\right]^{2} ; \\
& \omega_{0}^{S_{t}}=\omega_{0}^{0}\left(1-S_{t}\right)+\omega_{0}^{1} S_{t} .
\end{aligned}
$$

A constant variance model will be defined as the above process subject to the restriction of $\omega_{1}=0$. With respect to the jump component, we will make the following assumptions on both $\lambda_{t}$ and the volatility of the jump size, i.e.

$$
\lambda_{S_{t}}=\lambda_{0}\left(1-S_{t}\right)+\lambda_{1} S_{t}
$$

and

$$
\psi_{S_{t}}=\psi_{0}\left(1-S_{t}\right)+\psi_{1} S_{t}
$$

Note that we assume that $\lambda_{t}$ is constant instead of being time-varying as shown in equation (10) in order to simplify the model. The same reason holds for the mean of the jump size that is supposed to be the same value across the different states. Here, we will adopt the specification of $\theta_{t}=\gamma\left(r_{t-1}-r_{t-1}^{*}\right)$. 
Section A.1 from the Appendix shows the construction of the likelihood function for this process.

\subsection{The conditional mean and variance}

The conditional mean of our RS model with jumps is

$$
E_{t-1}\left[\Delta r_{t}\right]=p_{0, t} \tilde{\mu}_{0, t}+\left(1-p_{0, t}\right) \tilde{\mu}_{1, t}
$$

where $p_{0, t} \equiv P\left[S_{t}=0 \mid \Phi_{t-1}\right]$ and $1-p_{0, t}$ are the ex-ante probabilities of states 0 and $1-$ see section A.1 from Appendix - and $\tilde{\mu}_{S_{t}, t}$ is the conditional mean of state $S_{t}$ at date $t$ :

$$
\tilde{\mu}_{S_{t}, t}=\mu_{S_{t}, t}+\lambda_{S_{t}} \theta_{t}
$$

The conditional variance is obtained as

$$
\begin{aligned}
V_{t-1}\left(\Delta r_{t}\right)= & E_{t-1}\left(\Delta r_{t}^{2}\right)-E_{t-1}\left(\Delta r_{t}\right)^{2} \\
& =p_{0, t}\left(h_{0, t}+\tilde{\mu}_{0, t}^{2}\right)+\left(1-p_{0, t}\right)\left(h_{1, t}+\tilde{\mu}_{1, t}^{2}\right)-\left[p_{0, t} \tilde{\mu}_{0, t}+\left(1-p_{0, t}\right) \tilde{\mu}_{1, t}\right]^{2}
\end{aligned}
$$

where $h_{S_{t}, t}$ is the conditional variance of state $S_{t}$ at date $t$ :

$$
h_{S_{t}, t}=\sigma_{S_{t}, t}^{2}+\lambda_{S_{t}}\left[\psi_{S_{t}}^{2}+\left(1-\lambda_{S_{t}}\right) \theta_{t}^{2}\right] \text {. }
$$

\subsection{Time varying transition probabilities}

We will also implement an augmentation of the above RS but a time-varying structure for the transition probabilities in equation (11) - denoted as $p_{i i, t}$ where $i=0,1$ - is allowed. We will assume two alternative specifications: 


$$
p_{i i, t}=\frac{\exp \left(\beta_{0}^{i}+\beta_{1}^{i}\left|r_{t-1}-r_{t-1}^{*}\right|\right)}{1+\exp \left(\beta_{0}^{i}+\beta_{1}^{i}\left|r_{t-1}-r_{t-1}^{*}\right|\right)}
$$

and

$$
p_{i i, t}=\frac{\exp \left(\beta_{0}^{i}+\beta_{1}^{i}\left|r_{t-1}-r_{t-1}^{*}\right|+\beta_{2}^{i} D_{t}^{m p}+\beta_{3}^{i} D_{t}^{m}\right)}{1+\exp \left(\beta_{0}^{i}+\beta_{1}^{i}\left|r_{t-1}-r_{t-1}^{*}\right|+\beta_{2}^{i} D_{t}^{m p}+\beta_{3}^{i} D_{t}^{m}\right)}
$$

where $p_{i i, t} \equiv P\left[S_{t}=i \mid S_{t-1}=i\right]$ such that $i=0,1^{12}$. Equation (20) depends on the size of the spread at time $t-1$. Note that the behavior of the spread time series will depend mainly on the dynamics of the EONIA rate rather than the evolution of the official rate which hardly changes as it is shown in Figure 1. This model nests the constant transition probability, or equation (11), when $\beta_{1}^{0}=\beta_{1}^{1}=0$. Meanwhile, equation (21), which nests (20) when $\beta_{2}^{i}=\beta_{3}^{i}=0$, also incorporates the well known seasonal pattern captured by both the maintenance period (mp) and the end of the month (m) effect. Equation (21) nests the constant transition probability when $\beta_{1}^{i}=\beta_{2}^{i}=\beta_{3}^{i}=0$.

\subsection{Results}

Table 10 exhibits the estimates of the RS model with constant transition probabilities under alternative specifications of both the conditional mean $\mu_{S_{t}, t}$ (single or double mean reversion) and conditional variance $\sigma_{S_{t}, t}^{2}$ (constant variance or $\mathrm{ARCH}$ (1) structure). A total of five models are estimated: model I is a Gaussian process with ARCH (1) structure and single mean reversion in each state. Remark on the other models in Table 10, and also in Table 11 as we will comment in short, that states 0 and 1 will always be characterised by a Poisson-Gaussian process under alternative specifications of both the mean reversion and

\footnotetext{
12 Note that $p_{i i, t} \equiv P\left[S_{t}=i \mid S_{t-1}=i\right]$ is an abbreviated form. Equation (20) must be really expressed as $p_{i i, t} \equiv P\left[S_{t}=i \mid S_{t-1}=i, \Gamma_{t-1}\right]$ where $\Gamma_{t-1}$ is an information set that affects the likelihood of regime switches. In this case, $\Gamma_{t-1}=\left|r_{t-1}-r_{t-1}^{*}\right|$. Meanwhile, in equation (21), $p_{i, t} \equiv P\left[S_{t}=i \mid S_{t-1}=i, \Gamma_{t-1}, \Upsilon_{t}\right]$ where $\Upsilon_{t}$ is a different information set at time $t$ containing the seasonal components $D_{t}^{m p}$ and $D_{t}^{m}$ which are known at time $t-1$.
} 
the conditional variance. Model II shows single mean reversion and constant variance, model III presents single mean reversion but an ARCH (1) structure, model IV shows a double mean reversion and constant variance and finally, a double mean reversion but an ARCH (1) structure is the specification of model V. Models III and V nest models II and IV when there is no ARCH effect, i.e. $\omega_{1}=0$, respectively. Note also that model IV nests model II when there is no a double mean reversion, i.e. $\alpha_{1}^{S_{t}}=\alpha_{2}^{S_{t}}$, and so on. The models in Table 11, denoted as VI to IX, are characterised as follows: models VI and VII are similar to models II and III respectively, but now with a time varying transition probability $p_{i i, t}$ defined in (20); meanwhile models VIII and IX are the same as models II and III respectively but $p_{i i, t}$ is driven by equation (21). To ensure that we identify the global maximum we have reestimated each model with different sets of starting values.

Looking at tables 10 and 11, we can appreciate some features: first, modeling the EONIA rate under a regime-switching $\mathrm{ARCH}$ process but without the introduction of the jump component in each state (model I) leads to the lowest values of both the likelihood function and SIC. For instance, model III which is the same as model I - that is, single mean reversion and ARCH (1) structure - but with a Poisson-Gaussian specification shows a SIC value of 2223.65 against 2128.49 for model I. This large difference about more than one hundred is due to the lack of the jump component in model I.

Second, there is only ARCH (1) effect - the coefficient $\omega_{1}$ is significant -in model I. It also holds that the magnitude of $\omega_{1}$ decreases sharply going from model I to another model. For example, $\omega_{1}=0.0056$ in model III against $\omega_{1}=0.4391$ in model I. The reason is once we plug the jump component into each state in model I, and so we obtain model III, the ARCH contribution in the variance of each state becomes less important. If we take the conditional variance of state $S_{t}$ at date $t$ denoted as $h_{S_{t}, t}$ - see equation (19) - then we can observe that $h_{S_{t}, t}$ for model I is equal to the $\mathrm{ARCH}(1)$ component $\sigma_{S_{t}, t}^{2}$ defined in equation (14) while $h_{S_{t}, t}$ for model III is the sum of the ARCH effect and the jump effect. Note that this last component is $\lambda_{S_{t}}\left[\psi_{S_{t}}^{2}+\left(1-\lambda_{S_{t}}\right) \theta_{t}^{2}\right]$. 
Table 10. Poisson-Gaussian model with a regime-switching process (constant transition probabilities)

Daily data of EONIA rate covering the period January 1999 to December 2003. This table contains Poisson-Gaussian models with a regime-switching process and constant transition probabilities under different specifications for the conditional mean (single or double mean reversion) and conditional variance (constant or ARCH (1) variance). The system switches between two regimes, $S_{t}=0,1$, where each state follows a Poisson-Gaussian process except model I (Gaussian process in each state). In each regime, the parameters for the conditional mean and variance with no jumps, denoted as $\mu_{s, t}$ and $\sigma_{s, t}^{2}$ respectively; the jump intensity $\lambda_{s}$; and the jump volatility $\psi_{s_{t}}$ are varied. The Markov chain is defined by the switching probabilities in equation (11). The ARCH (1) specification is $\sigma_{S_{t}, t}^{2}=\omega_{0}^{S_{t}}+\omega_{1}\left[\Delta r_{t-1}-E_{t-2}\left(\Delta r_{t-1}\right)\right]^{2}$ where $\omega_{0}^{S_{t}}=\omega_{0}^{0}\left(1-S_{t}\right)+\omega_{0}^{1} S_{t}$. The constant variance is defined as $\sigma_{S_{t}, t}^{2}=\omega_{0}^{S_{t}}$, i.e. the above process subject to $\omega_{1}=0$.

\begin{tabular}{|c|c|c|c|c|c|c|}
\hline & & \multicolumn{3}{|c|}{ Single mean reversion } & \multicolumn{2}{|c|}{ Double mean reversion } \\
\hline \multirow[t]{3}{*}{ State } & Parameters & & $\begin{array}{l}\mu_{S_{t, t}}=\alpha^{S_{t}}\left(r_{t-1}-r_{t-1}^{*}\right) \\
\alpha^{S_{t}}=\alpha^{0}\left(1-S_{t}\right)+c\end{array}$ & & $\begin{array}{l}\mu_{S_{t}, t}=\alpha_{1}^{S_{t}}\left(r_{t-1}-r_{t-1}^{*}\right. \\
\alpha_{k}^{S_{t}}=\alpha_{k}^{0}\left(1-S_{t}\right)+c\end{array}$ & $\begin{array}{l}S_{t}\left(r_{t-1}-r_{t-1}^{*}\right)^{-} \\
\text {where } k=1,2\end{array}$ \\
\hline & & Gaussian & \multicolumn{4}{|c|}{ Poisson-Gaussian } \\
\hline & & $\begin{array}{c}\text { (I) } \\
\text { ARCH (1) }\end{array}$ & $\begin{array}{c}\text { (II) } \\
\text { Constant variance }\end{array}$ & $\begin{array}{c}\text { (III) } \\
\text { ARCH (1) }\end{array}$ & $\begin{array}{c}\text { (IV) } \\
\text { Constant variance }\end{array}$ & $\begin{array}{c}\mathrm{V}) \\
\mathrm{ARCH}(1)\end{array}$ \\
\hline 0 & $\alpha^{0}$ & $\begin{array}{l}-0.0421 \\
(-3.622) \\
\end{array}$ & $\begin{array}{r}-0.0182 \\
(-0.993) \\
\end{array}$ & $\begin{array}{l}-0.0273 \\
(-5.325) \\
\end{array}$ & ----- & ----- \\
\hline 1 & $\alpha^{1}$ & $\begin{array}{c}-0.5452 \\
(-10.343) \\
\end{array}$ & $\begin{array}{l}0.0313 \\
(0.817) \\
\end{array}$ & $\begin{array}{l}0.0875 \\
(2.286)\end{array}$ & ------ & ------ \\
\hline 0 & $\alpha_{1}^{0}$ & ----- & ----- & ----- & $\begin{array}{l}-0.0206 \\
(-1.542)\end{array}$ & $\begin{array}{l}-0.0274 \\
(-4.634)\end{array}$ \\
\hline 1 & $\alpha_{1}^{1}$ & ------ & ------ & ------ & $\begin{array}{l}0.0234 \\
(0.968)\end{array}$ & $\begin{array}{l}0.0578 \\
(0.988)\end{array}$ \\
\hline 0 & $\alpha_{2}^{0}$ & ----- & ----- & ------ & $\begin{array}{l}-1.0909 \\
(-123.61)\end{array}$ & $\begin{array}{c}-1.0855 \\
(-43.054)\end{array}$ \\
\hline 1 & $\alpha_{2}^{1}$ & ------ & ------ & ------ & $\begin{array}{l}0.1347 \\
(1.732) \\
\end{array}$ & $\begin{array}{l}0.1709 \\
(2.168)\end{array}$ \\
\hline 0 & $\omega_{0}^{0}$ & $\begin{array}{c}0.00004 \\
(5.379)\end{array}$ & $\begin{array}{c}0.00003 \\
(7.267) \\
\end{array}$ & $\begin{array}{c}0.00003 \\
(6.786) \\
\end{array}$ & $\begin{array}{c}0.00004 \\
(6.669)\end{array}$ & $\begin{array}{c}0.00003 \\
(7.042) \\
\end{array}$ \\
\hline 1 & $\omega_{0}^{1}$ & $\begin{array}{l}0.0365 \\
(6.946)\end{array}$ & $\begin{array}{l}0.0032 \\
(3.760)\end{array}$ & $\begin{array}{l}0.0033 \\
(5.120)\end{array}$ & $\begin{array}{l}0.0028 \\
(4.599)\end{array}$ & $\begin{array}{l}0.0030 \\
(4.136)\end{array}$ \\
\hline both & $\omega_{1}$ & $\begin{array}{l}0.4391 \\
(3.304) \\
\end{array}$ & ----- & $\begin{array}{l}0.0056 \\
(1.380) \\
\end{array}$ & ----- & $\begin{array}{l}0.0046 \\
(0.799) \\
\end{array}$ \\
\hline both & $\gamma$ & ---- & $\begin{array}{l}-1.2783 \\
(-3.598)\end{array}$ & $\begin{array}{l}-1.8410 \\
(-7.547)\end{array}$ & $\begin{array}{l}-1.2612 \\
(-3.246)\end{array}$ & $\begin{array}{l}-1.6295 \\
(-3.269)\end{array}$ \\
\hline 0 & $\psi_{0}$ & ----- & $\begin{array}{l}0.0058 \\
(1.951)\end{array}$ & $\begin{array}{l}0.0068 \\
(2.325)\end{array}$ & $\begin{array}{l}0.0064 \\
(1.135)\end{array}$ & $\begin{array}{l}0.0072 \\
(2.714)\end{array}$ \\
\hline 1 & $\psi_{1}$ & ------ & $\begin{array}{c}0.2643 \\
(17.918)\end{array}$ & $\begin{array}{c}0.2519 \\
(15.092)\end{array}$ & $\begin{array}{c}0.2646 \\
(17.238)\end{array}$ & $\begin{array}{c}0.2521 \\
(14.020)\end{array}$ \\
\hline 0 & $\lambda_{0}$ & ------ & $\begin{array}{l}0.1393 \\
(3.431)\end{array}$ & $\begin{array}{l}0.1127 \\
(5.310)\end{array}$ & $\begin{array}{l}0.1370 \\
(4.026)\end{array}$ & $\begin{array}{l}0.1292 \\
(4.430)\end{array}$ \\
\hline 1 & $\lambda_{1}$ & ------ & $\begin{array}{c}0.4079 \\
(10.877)\end{array}$ & $\begin{array}{c}0.4146 \\
(10.480)\end{array}$ & $\begin{array}{l}0.3817 \\
(9.316)\end{array}$ & $\begin{array}{l}0.3739 \\
(8.283)\end{array}$ \\
\hline 0 & $p_{00}$ & $\begin{array}{c}0.8339 \\
(41.623)\end{array}$ & $\begin{array}{c}0.8045 \\
(43.378)\end{array}$ & $\begin{array}{c}0.8016 \\
(47.075)\end{array}$ & $\begin{array}{c}0.8124 \\
(47.531)\end{array}$ & $\begin{array}{c}0.8095 \\
(48.605)\end{array}$ \\
\hline 1 & $p_{11}$ & $\begin{array}{c}0.5901 \\
(12.661) \\
\end{array}$ & $\begin{array}{c}0.7850 \\
(29.590)\end{array}$ & $\begin{array}{c}0.7703 \\
(32.667)\end{array}$ & $\begin{array}{c}0.8001 \\
(34.149)\end{array}$ & $\begin{array}{c}0.7732 \\
(22.023)\end{array}$ \\
\hline & $\begin{array}{c}\text { Log-L } \\
\text { Function }\end{array}$ & 2153.59 & 2255.01 & 2266.70 & 2273.10 & 2299.43 \\
\hline & $\mathrm{SIC}$ & 2128.49 & 2215.56 & 2223.65 & 2226.48 & 2249.22 \\
\hline
\end{tabular}

Estimation is undertaken using maximum-likelihood. Heteroskedasticity-Consistent t-statistics are in parentheses. 
Table 11. Poisson-Gaussian models with a regime-switching process (time varying transition probabilities)

Daily data of EONIA rate covering the period January 1999 to December 2003. This table contains Poisson-Gaussian models with a regime-switching process and time varying transition probabilities. The system switches between two regimes, $S_{t}=0,1$, where each state follows a Poisson-Gaussian process. In each regime, the parameters for the conditional mean and variance with no jumps, denoted as $\mu_{S_{t}, t}$ and $\sigma_{S_{t}, t}^{2}$ respectively; the jump intensity $\lambda_{S_{t}}$; and the jump volatility $\psi_{S_{t}}$ are varied. The Markov chain is defined by the switching probabilities $p_{i i, t} \equiv P\left[S_{t}=i \mid S_{t-1}=i\right] \quad$ where $i=0,1 \quad$ and $\quad p_{i i, t}=\varphi\left(x_{t}\right) \quad$ where $\quad \varphi\left(x_{t}\right)=\exp \left(x_{t}\right) /\left(1+\exp \left(x_{t}\right)\right)$. The specification of $\mu_{S_{t}, t}$ and $\sigma_{S_{t}, t}^{2}$ are respectively:

$$
\begin{aligned}
& \mu_{s_{t, t}}=\alpha^{S_{t}}\left(r_{t-1}-r_{t-1}^{*}\right) \quad \text { where } \alpha^{S_{t}}=\alpha^{0}\left(1-S_{t}\right)+\alpha^{1} S_{t} \\
& \sigma_{S_{t, t}}^{2}=\omega_{0}^{S_{t}}+\omega_{1}\left[\Delta r_{t-1}-E_{t-2}\left(\Delta r_{t-1}\right)\right]^{2} \quad \text { where } \quad \omega_{0}^{S_{t}}=\omega_{0}^{0}\left(1-S_{t}\right)+\omega_{0}^{1} S_{t} ; \quad\left(\omega_{1}=0: \text { constant variance }\right)
\end{aligned}
$$

\begin{tabular}{|c|c|c|c|c|c|}
\hline & & \multicolumn{2}{|c|}{$p_{i i, t}=\varphi\left(\beta_{0}^{i}+\beta_{1}^{i}\left|r_{t-1}-r_{t-1}^{*}\right|\right)$} & \multicolumn{2}{|c|}{$p_{i i, t}=\varphi\left(\beta_{0}^{i}+\beta_{1}^{i}\left|r_{t-1}-r_{t-1}^{*}\right|+\beta_{2}^{i} D_{t}^{m p}+\beta_{3}^{i} D_{t}^{m}\right)$} \\
\hline State & Parameters & (VI) Constant variance & (VII) ARCH (1) & (VIII) Constant variance & (IX) ARCH (1) \\
\hline 0 & $\alpha^{0}$ & $\begin{array}{l}-0.0236 \\
(-3.036)\end{array}$ & $\begin{array}{l}-0.0263 \\
(-4.981)\end{array}$ & $\begin{array}{l}-0.0311 \\
(-4.406)\end{array}$ & $\begin{array}{l}-0.0311 \\
(-5.450)\end{array}$ \\
\hline 1 & $\alpha^{1}$ & $\begin{array}{l}0.0215 \\
(0.538)\end{array}$ & $\begin{array}{l}0.0479 \\
(1.399)\end{array}$ & $\begin{array}{l}0.0376 \\
(1.108)\end{array}$ & $\begin{array}{l}0.0491 \\
(1.371)\end{array}$ \\
\hline 0 & $\omega_{0}^{0}$ & $\begin{array}{l}0.00004 \\
(7.232)\end{array}$ & $\begin{array}{l}0.00003 \\
(7.116) \\
\end{array}$ & $\begin{array}{l}0.00004 \\
(6.495) \\
\end{array}$ & $\begin{array}{l}0.00003 \\
(5.173) \\
\end{array}$ \\
\hline 1 & $\omega_{0}^{1}$ & $\begin{array}{l}0.0033 \\
(3.110)\end{array}$ & $\begin{array}{l}0.0038 \\
(5.668)\end{array}$ & $\begin{array}{l}0.0044 \\
(4.312)\end{array}$ & $\begin{array}{l}0.0043 \\
(4.734)\end{array}$ \\
\hline Both & $\omega_{1}$ & ------ & $\begin{array}{l}0.0047 \\
(1.345)\end{array}$ & ------ & $\begin{array}{l}0.0074 \\
(0.710)\end{array}$ \\
\hline Both & $\gamma$ & $\begin{array}{l}-1.2727 \\
(-2.655)\end{array}$ & $\begin{array}{l}-1.7986 \\
(-5.486)\end{array}$ & $\begin{array}{l}-1.1190 \\
(-3.322)\end{array}$ & $\begin{array}{l}-1.5556 \\
(-5.120)\end{array}$ \\
\hline 0 & $\psi_{0}$ & $\begin{array}{l}0.0061 \\
(2.006)\end{array}$ & $\begin{array}{l}0.0067 \\
(2.061)\end{array}$ & $\begin{array}{l}0.0241 \\
(4.266)\end{array}$ & $\begin{array}{l}0.0212 \\
(5.332)\end{array}$ \\
\hline 1 & $\psi_{1}$ & $\begin{array}{c}0.2667 \\
(18.770)\end{array}$ & $\begin{array}{c}0.2601 \\
(15.787)\end{array}$ & $\begin{array}{c}0.2654 \\
(17.838)\end{array}$ & $\begin{array}{c}0.2607 \\
(16.575)\end{array}$ \\
\hline 0 & $\lambda_{0}$ & $\begin{array}{l}0.1298 \\
(4.141)\end{array}$ & $\begin{array}{l}0.1135 \\
(6.637)\end{array}$ & $\begin{array}{l}0.1892 \\
(5.020)\end{array}$ & $\begin{array}{l}0.1762 \\
(6.137) \\
\end{array}$ \\
\hline 1 & $\lambda_{1}$ & $\begin{array}{l}0.4015 \\
(9.699) \\
\end{array}$ & $\begin{array}{l}0.3918 \\
(9.871) \\
\end{array}$ & $\begin{array}{l}0.4635 \\
(9.096) \\
\end{array}$ & $\begin{array}{l}0.4403 \\
(8.776) \\
\end{array}$ \\
\hline 0 & $\beta_{0}^{0}$ & $\begin{array}{l}1.4681 \\
(6.505)\end{array}$ & $\begin{array}{l}1.3792 \\
(8.253)\end{array}$ & $\begin{array}{l}3.0148 \\
(7.968)\end{array}$ & $\begin{array}{l}2.8766 \\
(7.588)\end{array}$ \\
\hline 1 & $\beta_{0}^{1}$ & $\begin{array}{l}0.0758 \\
(0.322)\end{array}$ & $\begin{array}{l}0.1176 \\
(0.495)\end{array}$ & $\begin{array}{l}-1.5327 \\
(-2.984)\end{array}$ & $\begin{array}{l}-1.3691 \\
(-2.700)\end{array}$ \\
\hline 0 & $\beta_{1}^{0}$ & $\begin{array}{l}-1.6935 \\
(-0.471)\end{array}$ & $\begin{array}{l}-0.0874 \\
(-0.040)\end{array}$ & $\begin{array}{l}-2.7050 \\
(-0.556)\end{array}$ & $\begin{array}{l}-3.1237 \\
(-0.730)\end{array}$ \\
\hline 1 & $\beta_{1}^{1}$ & $\begin{array}{l}9.9756 \\
(3.943)\end{array}$ & $\begin{array}{l}8.5354 \\
(4.415)\end{array}$ & $\begin{array}{l}16.0894 \\
(3.734)\end{array}$ & $\begin{array}{l}13.526 \\
(2.783)\end{array}$ \\
\hline 0 & $\beta_{2}^{0}$ & ------ & ------ & $\begin{array}{l}-2.8226 \\
(-8.046) \\
\end{array}$ & $\begin{array}{l}-2.6925 \\
(-8.001) \\
\end{array}$ \\
\hline 1 & $\beta_{2}^{1}$ & ------ & ------ & $\begin{array}{l}16.7859 \\
(0.917)\end{array}$ & $\begin{array}{c}19.142 \\
(0.3556)\end{array}$ \\
\hline 0 & $\beta_{3}^{0}$ & ------ & ------ & $\begin{array}{l}-2.8747 \\
(-7.788)\end{array}$ & $\begin{array}{l}-2.7674 \\
(-8.406)\end{array}$ \\
\hline 1 & $\beta_{3}^{1}$ & ------ & ------ & $\begin{array}{l}12.5492 \\
(0.427)\end{array}$ & $\begin{array}{l}11.525 \\
(2.559)\end{array}$ \\
\hline & $\begin{array}{c}\text { Log-Likelihood } \\
\text { Function }\end{array}$ & 2286.80 & 2293.21 & 2384.62 & 2393.14 \\
\hline & SIC & 2240.18 & 2243.00 & 2323.66 & 2328.59 \\
\hline
\end{tabular}

Estimation is undertaken using maximum-likelihood. Heteroskedasticity-Consistent t-statistics are in parentheses. 
Third, both variance components, i.e. $\omega_{0}^{S_{t}}$ from $\sigma_{S_{t}, t}^{2}$ (no jump) and $\psi_{S_{t}}^{2}$ (jump), are higher in state 1 than in state 0 . The intensity parameter, or the probability of occurring one jump, $\lambda_{S_{t}}$ is also higher in state 1 . This suggests that state 0 is a period of lower volatility than state 1 . In order to strengthen this result, a simple analysis based on the smoothed probability - see section A.2 in Appendix - with the criterion of $P\left[S_{t}=0 \mid \Phi_{T}\right]>0.5$ is established to identify the day $t$ as regime 0 and so, regime 1 for the case of the above probability lower or equal to 0.5 . Once we have set a regime to each day, we compute for instance the total conditional variance according to equation (18) for model IX - it makes the best scoring according to the SIC value - which is exhibited in Figure 3 and then, we split this sample into two parts corresponding each to states 0 and 1 respectively (state 0 includes 805 days against 495 days for state 1) and compute both the mean and the standard deviation to each subsample. The mean of the daily variance in state 0 is 0.009 with a standard deviation of 0.011 against a mean of 0.078 with a standard deviation of 0.111 in state 1 . Since the variation coefficient (standard deviation/mean) is rather the same for both samples, we can affirm that state 1 displays more volatility than state 0 .

Figure 3. Daily conditional variance (Regime switching with jumps)

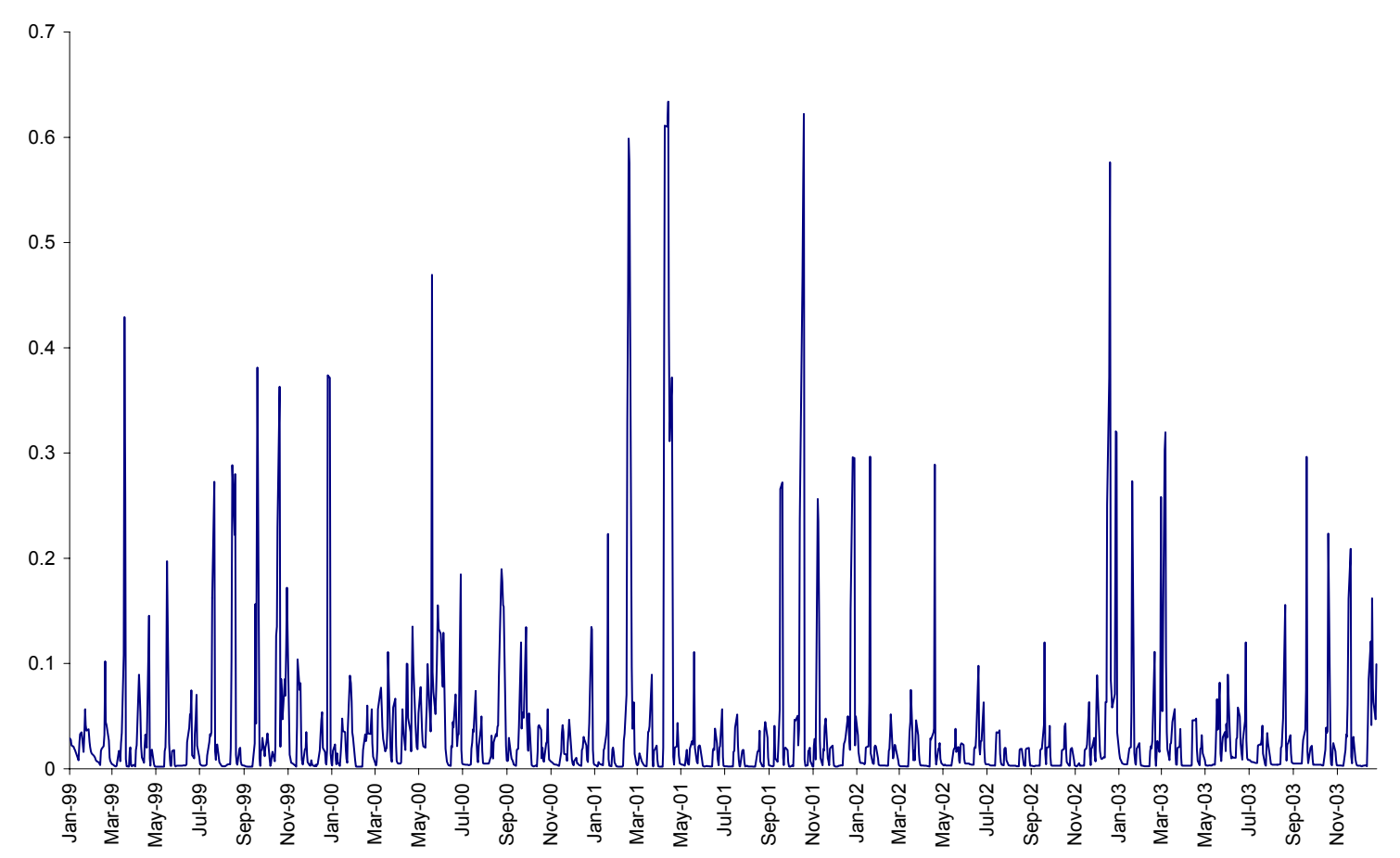


The next question we wonder why regime 1 is more volatile. We find that the percentage of days containing only those days for either the end of the maintenance period (mp) or the end of month $(\mathrm{m})$ tends to be higher in regime 1. It also holds that the percentage of days of the end of the maintenance period is always higher than the percentage of days of the end of the month in regime 1 in each model. Model IX shows an amount of $22.39 \%$ days in regime 0 corresponding to the total of both effects while a percentage of $36.16 \%$ in regime 1 . The $\mathrm{mp}(\mathrm{m})$ effect represents in regime 0 a size of $41.25 \%(68.33 \%)$ over the total days in the sample corresponding to the $\mathrm{mp}(\mathrm{m})$ effect. In short, since these magnitudes are rather the same across the different models, it means that $\mathrm{mp}(\mathrm{m})$ is higher (lower) in regime 1 but the size of both effects is higher in regime 1 . In section 5 it was shown that the highest variance average was reached during the end of the maintenance period while the end of month was higher than the rest of days (o). Again, this empirical fact might help to understand why regime 1 exhibits more volatility than regime 0 since both effects are more concentrated here. Finally, it is also prompt to understand why the probability of occurring a jump is higher in regime 1 since, as we mentioned in section $5, \lambda_{t}$ is higher for either mp or $\mathrm{m}$ than any other day (o) as Table 4 reports.

Fourth, Model I shows a significant mean reversion in both states. This result is contrary to the evidence observed in the rest of the models in tables 10 and 11 characterised by a unique regime of mean reversion. Nevertheless, we will concentrate on the behavior of all models except model I since its SIC value is pretty worse than the others as we commented before due to the lack of modeling the jump effect in each state. In short, the misspecification of jumps leads to model I to exhibit mean reversion in the two states but the introduction of jumps makes a higher performance, according to the SIC value, and shows a pattern of mean reversion completely different in each state that is more in accordance with the empirical evidence shown by Gray (1996) and so forth. Specifically, the sign of the mean reversion (both single and double) is always negative in state 0 while it is positive in state 1 (except for model I). This evidence suggests that the EONIA rate is mean reverting to the official rate in state 0 characterised by a period of low volatility while the mean reversion does not guarantee in state 1 . It is held that the mean reversion (state 0 ) tends to be more significant under the ARCH (1) structure. Indeed, the mean reversion (single and double) is always significant, except for models II and IV (constant variance). It is also verified that the double mean reversion beats the single one. 
An LRT statistic, which is distributed as $\chi^{2}(2)$ under the null or restricted model, is 36.18 (p-value equals zero) and hence, a rejection of model II (single mean reversion) against model IV (double mean reversion). The same conclusion maintains under the ARCH (1) structure, i.e. model III is rejected in favor of model V.

Last but not least, the models under time varying transition probabilities improve the performance ${ }^{13}$ in the sense of higher SIC values. An LRT statistic rejects the constant probability model II in favor of model VI. The LRT, which is also distributed $\chi^{2}(2)$, is 63.58 and its p-value equals zero. We arrive at the same conclusion when the null and the alternative hypotheses are models III and VII respectively. Finally, an LRT statistic rejects model VI (model VII) in favor of model VIII (model IX). The LRT statistic, that is distributed $\chi^{2}(4)$, is 195.64 (199.86) and its p-value is zero (zero) again. Note also that most of the parameters of the time varying transition probability in equation (21) concerning models VIII and IX are significant.

Finally, we must remark that our evidence on both low volatility and mean reversion in state 0 and the opposite in state 1 is different with respect to the other works cited previously. It is worth to emphasize that all those papers employ interest rate series having either a weekly or monthly frequency while we use daily data. The selection of a higher frequency might be a possible reason why this study brings out a different result. A possible answer to our results can be found in the analysis of the sign of the parameters which define $p_{i i, t}$ in the equations (20) and (21). If we take model VI or VII and consider an increase in the size of the spread $\left|r_{t-1}-r_{t-1}^{*}\right|$. Since $\beta_{1}^{0}$ is negative (state 0 ), the probability of staying in this regime decreases as the size of the spread increases. Conversely, since $\beta_{1}^{1}$ is positive (state 1 ) the probability of staying in this regime increases as the size of the spread increases. Therefore, when the size of the spread raises - mainly due to a rise in the EONIA rate - the probability of staying or switching to regime 1 increases. In short, high level of interest rates takes place in regime 1 in addition to both a higher probability of occurring jumps and the failure of holding the mean reversion in this

\footnotetext{
${ }^{13}$ We do not estimate RS under double mean reversion with time varying transition probabilities. The reason is based on the large number of parameters we had to estimate.
} 
state. Now, if we consider model VIII or IX, the same conclusion holds about the sensitivity of $p_{i i, t}$ to changes in $\left|r_{t-1}-r_{t-1}^{*}\right|$, i.e. $\beta_{1}^{0}<0$ and $\beta_{1}^{1}>0$. The sensitivity of the end of the maintenance period or the end of month in regime 0 is measured through the parameters $\beta_{2}^{0}$ and $\beta_{3}^{0}$ respectively and they are negative while they show the opposite sign in regime 1. This evidence reinforces our suspicion or hypothesis that if tomorrow $D_{t}^{m p}=1$ or $D_{t}^{m}=1$ then the probability of staying or switching to regime 1 increases.

\subsection{Regime classification}

To measure the quality of the regime classification we use the regime classification measure (RCM) proposed by Ang and Bekaert (2002a), defined for two states as

$$
\mathrm{RCM}=400 \times \frac{1}{T} \sum_{t=1}^{T} P\left[S_{t}=0 \mid \Phi_{T}\right] \times P\left[S_{t}=1 \mid \Phi_{T}\right]
$$

where $P\left[S_{t}=i \mid \Phi_{T}\right]$ is the smoothed probability of state $i$ (see section A.2 in Appendix). $\mathrm{RCM}$ is between 0 and 100. A good regime classification is associated with low RCM statistic values. A value of 0 means perfect classification and a value of 100 implies no information about the regimes is revealed. It is shown that moving from constant to time varying transition probabilities produces an improvement. For example, consider the case of constant variance (ARCH structure) and single mean reversion: model II (model III) leads to a value of RCM equals 21.12 (23.78) which is higher than 19.79 (21.49) and 18.31 (19.45) for models VI (VII) and VIII (IX) respectively. It is inferred from these results that a better classification is obtained with time varying transition probabilities. It also holds that the constant variance specification beats the ARCH structure. Note that model I, the only specification which does not incorporate jumps in any state, scores the worst classification with a value of 25.53 under a single mean reversion structure. Finally, the regime classification shown by the double mean reversion is worse than the single mean reversion: the RCM values corresponding to models IV and V are 38.21 and 23.06 respectively. Contrary to the single mean reversion, the ARCH structure beats in this case. 


\section{Out-of-sample performance}

We estimate the parameters of a particular model over the in-sample period which are held fixed to compute a time series of conditional variances over the out-of-sample period. We repeat this process several times for alternative in-sample periods. Let $\varepsilon_{t}=\Delta r_{t}-E_{t-1}\left[\Delta r_{t}\right]$ denote the innovation to the interest rate process. We compare the conditional variance $E_{t-1}\left[\varepsilon_{t}^{2}\right]$ with the actual squared innovation $\varepsilon_{t}^{2}$. The difference between $\varepsilon_{t}^{2}$ and $E_{t-1}\left[\varepsilon_{t}^{2}\right]$ is the error denoted as $e_{t}$. We will use the following common statistics for our in-sample and out-of-sample performance: the root mean squared error (RMSE), the mean absolute error (MAE) and the $R^{2}$ measure from the regression with dependent and independent variables $\varepsilon_{t}^{2}$ and $E_{t-1}\left[\varepsilon_{t}^{2}\right]$ respectively and imposing an intercept of zero and a slope of one. So, $R^{2}$ becomes a direct measure of the goodness of fit of the variance forecast. Note that negative values of $R^{2}$ are possible. For a time series $e_{t}$, these statistics are defined as:

$$
\mathrm{RMSE}=\sqrt{\frac{1}{T} \sum_{t=1}^{T} e_{t}^{2}} ; \quad \mathrm{MAE}=\frac{1}{T} \sum_{t=1}^{T}\left|e_{t}\right| \quad \text { and } \quad R^{2}=1-\sum_{t=1}^{T} e_{t}^{2} / \sum_{t=1}^{T} \varepsilon_{t}^{4}
$$

Tables 12 and 13 exhibit the in-sample and out-of-sample tests for different periods respectively. We compare the performance of some models of both one regime and two regimes. From the first group, we select both the single and the double mean reverting $\mathrm{ARCH}$ (1)-Poisson-Gaussian processes where $\lambda_{t}$ is constant, which represent models 2 and 4 from Table 3, and the double mean reverting ARCH-Poisson-Gaussian but with an autoregressive time varying $\lambda_{t}$ defined in equation (10), i.e. model $\mathrm{B}$ in Table 6 . In the second group, we select the ARCH-Gaussian with shifting regime specification - or model I in Table 10 - and all constant variance Poisson-Gaussian with shifting regime specifications: models II and IV in Table 10 (constant transition probabilities) and models VI and VIII in Table 11 (time varying transition probabilities). To shorten, we do not report the case of the $\mathrm{ARCH}$ specification for the two regime models since the 
performance of each model is worse than the one nested under the constant variance specification.

With regard to the in-sample analysis: first, the best fit - according to either the lowest value of MAE or RMSE - is classified depending on the mean reversion (single or double). For the single mean reversion, we select model VIII under both statistics. Meanwhile, for the double mean reversion, we select model 4 under MAE but model IV under RMSE. Note that the MAE values are rather the same for models 4 and B. Nevertheless, under RMSE model B beats. Second, if we compare a double mean reversion against a single one, it occurs that under one regime the double mean reversion is always better under both statistics but it does not happen the same under two regimes. Indeed, model IV is sometimes better than models I and II under RMSE but it never wins under MAE. Note that this last comparison is made under constant transition probabilities. Finally, comparing one regime with two regimes it happens that model 4 beats under MAE but model VIII beats under RMSE. There is also an improvement going from constant to time varying transition probability models, mainly because of the introduction of the dummy variables $D_{t}^{m p}$ and $D_{t}^{m}$ but not $\left|r_{t-1}-r_{t-1}^{*}\right|$. Specifically, compare models II and VIII.

In relation to the $R^{2}$ measure, note that it is always positive under any two regime specification reaching the best in-sample forecasting under model VIII. Nevertheless, under one regime specification $R^{2}$ is always negative for models 2 and 4 but it is always positive for model B. The $R^{2}$ values for model B are higher than those of model I except for the first period, that spans from January 1, 1999 to December 30, 2003, but they score the worst performance respecting the remaining two regime models. Negative values of $R^{2}$ occur when the in-sample daily variance forecast is much higher than the actual daily variance measured through $\varepsilon_{t}^{2}$. Hence, models 2 and 4 tend to an in-sample forecast over the actual variance.

With respect to the out-of-sample analysis: first, the best forecast - again according to either the lowest value of MAE or RMSE - is classified depending on the mean reversion (single or double). For the single mean reversion, we select model 2 under MAE 
Table 12. In-sample specification tests

Daily data of EONIA rate covering the period January 1999 to March 2005, a total of 1628 observations. This table contains mean absolute errors (MAE), root mean squared errors (RMSE) where the error $e_{t}$ is the difference between the actual daily variance $\left(\varepsilon_{t}^{2}\right.$ where $\left.\varepsilon_{t}=\Delta r_{t}-E_{t-1}\left[\Delta r_{t}\right]\right)$ and the conditional expected variance $\left(E_{t-1}\left[\varepsilon_{t}^{2}\right]\right)$ and the $R^{2}$ measure - with constant and slope equal zero and one respectively-defined as $R^{2}=1-\sum_{t=1}^{T} e_{t}^{2} / \sum_{t=1}^{T} \varepsilon_{t}^{4}$.

\begin{tabular}{|l|l|c|c|c|c|c|c|c|c|}
\hline & & \multicolumn{3}{|c|}{ One regime } & \multicolumn{4}{c|}{ Two regimes } \\
\hline $\begin{array}{c}\text { Period } \\
\text { (simple size) }\end{array}$ & Statistic & \multicolumn{2}{|c|}{ Table 3 } & Table 6 & \multicolumn{3}{c|}{ Table 10 } & \multicolumn{2}{c|}{ Table 11 } \\
\hline & & Model 2 & Model 4 & Model B & Model I & Model II & Model IV & Model VI & Model VIII \\
\hline Jan 1,1999- & MAE & 0.0270 & 0.0225 & 0.0226 & 0.0245 & 0.0259 & 0.0258 & 0.0264 & 0.0230 \\
Dec 30, 2003 & RMSE & 0.0777 & 0.0616 & 0.0587 & 0.0590 & 0.0514 & 0.0516 & 0.0534 & 0.0497 \\
$(1302)$ & $R^{2}$ & -0.1677 & -0.0734 & 0.1517 & 0.1555 & 0.2295 & 0.1872 & 0.2001 & 0.3116 \\
\hline Jan 1999- & MAE & 0.0263 & 0.0219 & 0.0220 & 0.0240 & 0.0250 & 0.0249 & 0.0259 & 0.0224 \\
Mar 3, 2004 & RMSE & 0.0766 & 0.0607 & 0.0581 & 0.0589 & 0.0502 & 0.0505 & 0.0530 & 0.0489 \\
$(1348)$ & $R^{2}$ & -0.1749 & -0.0809 & 0.1508 & 0.1405 & 0.2382 & 0.1910 & 0.1869 & 0.3094 \\
\hline Jan 1999- & MAE & 0.0263 & 0.0219 & 0.0219 & 0.0239 & 0.0248 & 0.0244 & 0.0256 & 0.0220 \\
May 5, 2004 & RMSE & 0.0770 & 0.0611 & 0.0583 & 0.0590 & 0.0501 & 0.0494 & 0.0525 & 0.0484 \\
$(1393)$ & $R^{2}$ & -0.1851 & -0.0772 & 0.1563 & 0.1407 & 0.2408 & 0.2102 & 0.1985 & 0.3171 \\
\hline Jan 1,1999- & MAE & 0.0257 & 0.0215 & 0.0216 & 0.0233 & 0.0243 & 0.0239 & 0.0246 & 0.0214 \\
Jun 30, 2004 & RMSE & 0.0758 & 0.0603 & 0.0578 & 0.0578 & 0.0493 & 0.0492 & 0.0508 & 0.0475 \\
$(1433)$ & $R^{2}$ & -0.1779 & -0.0773 & 0.1589 & 0.1504 & 0.2460 & 0.2126 & 0.2261 & 0.3245 \\
\hline Jan 1, 1999- & MAE & 0.0254 & 0.0214 & 0.0214 & 0.0230 & 0.0237 & 0.0234 & 0.0239 & 0.0208 \\
Sep 1, 2004 & RMSE & 0.0753 & 0.0601 & 0.0575 & 0.0575 & 0.0484 & 0.0484 & 0.0496 & 0.0463 \\
$(1478)$ & $R^{2}$ & -0.1852 & -0.0781 & 0.1584 & 0.1455 & 0.2558 & 0.2208 & 0.2415 & 0.3344 \\
\hline Jan 1, 1999- & MAE & 0.0252 & 0.0213 & 0.0214 & 0.0231 & 0.0236 & 0.0234 & 0.0236 & 0.0207 \\
Nov 3, 2004 & RMSE & 0.0749 & 0.0602 & 0.0580 & 0.0581 & 0.0482 & 0.0484 & 0.0491 & 0.0460 \\
$(1523)$ & $R^{2}$ & -0.1671 & -0.0686 & 0.1579 & 0.1388 & 0.2608 & 0.2243 & 0.2532 & 0.3381 \\
\hline Jan 1, 1999- & MAE & 0.0244 & 0.0206 & 0.0207 & 0.0225 & 0.0236 & 0.0234 & 0.0235 & 0.0204 \\
Jan 5, 2005 & RMSE & 0.0735 & 0.0592 & 0.0568 & 0.0578 & 0.0489 & 0.0488 & 0.0493 & 0.0458 \\
$(1568)$ & $R^{2}$ & -0.1564 & -0.0689 & 0.1563 & 0.1313 & 0.2212 & 0.1908 & 0.2316 & 0.3286 \\
\hline
\end{tabular}

except for the first two periods where model I scores the highest performance. Nevertheless, we choose model I under RMSE except for the last two periods where model 2 is the best. For the double mean reversion, we select model 4 under MAE but model IV under RMSE except for the last two periods where model 4 is the winner again. If we compare models 4 and B, it holds that under RMSE model B beats model 4 except for the last two periods. Second, if we compare a double mean reversion against a single one, it occurs that under one regime model 2 is always better under MAE. Meanwhile, under RMSE model B hits except for the last two periods where model 2 beats. For two regimes, model $\mathrm{I}$ is the winner under MAE but there is a trade-off between models I and IV depending on the selected period under RMSE. Again, this comparison is under constant 
transition probabilities. Finally, comparing one regime against two regimes it happens that model I beats under MAE until July 1, 2004 and afterward model 2 beats. Models 4, I and IV beat depending on the selected period under RMSE though the two regime models is better in most of the periods. Contrary to the in-sample analysis, there is no an improvement going from constant to time varying transition probability models.

The $R^{2}$ measure is positive for all the periods except the last two ones. For the case of $R^{2}>0$, model I scores the highest value. $R^{2}$ is also negative for the period going from September 2, 2004 to March 30, 2005 but only for model 2. The evidence of $R^{2}<0$ in the last two periods suggests that all models tend to forecast systematically daily variances higher that the actual variances. Note that this over prediction is lower for model 2.

\section{Conclusions}

This paper analyses the daily time-series of the Euro overnight interest rate (EONIA). We conclude that jumps are an essential component of modeling EONIA. The different models we implement contribute to a much better improvement in-sample fit once jumps are considered. Three effects such as the end of maintenance period effect, the calendar effect and the meeting effect show evidence of the existence of jumps. These effects have been considered for modeling jumps and specifically, the jump intensity or the ex-ante probability of occurring one jump. We find that both the end of maintenance period effect and the calendar effect cause greater jumps than the effect by the meetings of the Governing Council of the ECB. The lowest jump intensity corresponding to the days on which none of the effects occur leads to the conclusion that the main causes of the jumps are covered by our model. It also occurs that the persistence of the ARCH effects tend to decrease with the introduction of jumps.

The ECB's aim of keeping the overnight rate next to the official rate seems to sustain given the high level of reversion that empirical evidence suggests. We have analysed whether the speed of reversion is different when EONIA is higher than the official rate to when it is lower. It suggests that reversion is much greater in the latter case. In short, a two mean reversion specification has been implemented giving better results than a one mean reversion process. We have also tested the existence of overreaction in the 
Table 13. Out-of-sample specification tests

Daily data of EONIA rate covering the period January 1999 to March 2005, a total of 1628 observations. This table contains mean absolute errors (MAE), root mean squared errors (RMSE) where the error $e_{t}$ is the difference between the actual daily variance $\left(\varepsilon_{t}^{2}\right.$ where $\left.\varepsilon_{t}=\Delta r_{t}-E_{t-1}\left[\Delta r_{t}\right]\right)$ and the conditional expected variance $\left(E_{t-1}\left[\varepsilon_{t}^{2}\right]\right)$ and the $R^{2}$ measure - with constant and slope equal zero and one respectively-defined as $R^{2}=1-\sum_{t=1}^{T} e_{t}^{2} / \sum_{t=1}^{T} \varepsilon_{t}^{4}$.

\begin{tabular}{|l|l|c|c|c|c|c|c|c|c|}
\hline & & \multicolumn{3}{|c|}{ One regime } & \multicolumn{4}{c|}{ Two regimes } \\
\hline $\begin{array}{c}\text { Period } \\
\text { (simple size) }\end{array}$ & Statistic & \multicolumn{2}{|c|}{ Table 3 } & Table 6 & \multicolumn{3}{c|}{ Table 10 } & \multicolumn{2}{c|}{ Table 11 } \\
\hline & & Model 2 & Model 4 & Model B & Model I & Model II & Model IV & Model VI & Model VIII \\
\hline Dec 31,2003- & MAE & 0.0130 & 0.0131 & 0.0148 & 0.0126 & 0.0149 & 0.0140 & 0.0147 & 0.0130 \\
Mar 30, 2005 & RMSE & 0.0398 & 0.0398 & 0.0392 & 0.0284 & 0.0301 & 0.0291 & 0.0318 & 0.0312 \\
(326) & $R^{2}$ & 0.0591 & 0.0675 & 0.2081 & 0.3361 & 0.1933 & 0.2384 & 0.1036 & 0.1622 \\
\hline Mar 4, 2004- & MAE & 0.0135 & 0.0135 & 0.0153 & 0.0128 & 0.0151 & 0.0142 & 0.0151 & 0.0135 \\
Mar 30, 2005 & RMSE & 0.0427 & 0.0426 & 0.0417 & 0.0303 & 0.0316 & 0.0307 & 0.0340 & 0.0329 \\
$(280)$ & $R^{2}$ & 0.0651 & 0.0733 & 0.2233 & 0.3543 & 0.2293 & 0.2672 & 0.1166 & 0.1892 \\
\hline May 6, 2004- & MAE & 0.0112 & 0.0113 & 0.0134 & 0.0118 & 0.0140 & 0.0132 & 0.0140 & 0.0126 \\
Mar 30, 2005 & RMSE & 0.0360 & 0.0357 & 0.0350 & 0.0258 & 0.0267 & 0.0256 & 0.0284 & 0.0277 \\
$(235)$ & $R^{2}$ & 0.0190 & 0.0321 & 0.1866 & 0.3149 & 0.1865 & 0.2397 & 0.0840 & 0.1489 \\
\hline Jul 1, 2004- & MAE & 0.0111 & 0.0113 & 0.0139 & 0.0118 & 0.0144 & 0.0135 & 0.0144 & 0.0131 \\
Mar 30, 2005 & RMSE & 0.0386 & 0.0385 & 0.0379 & 0.0273 & 0.0285 & 0.0273 & 0.0300 & 0.0295 \\
$(195)$ & $R^{2}$ & 0.0188 & 0.0401 & 0.1929 & 0.3274 & 0.1943 & 0.2529 & 0.1153 & 0.1648 \\
\hline Sep 2, 2004- & MAE & 0.0102 & 0.0104 & 0.0133 & 0.0112 & 0.0139 & 0.0131 & 0.0139 & 0.0124 \\
Mar 30, 2005 & RMSE & 0.0392 & 0.0389 & 0.0389 & 0.0263 & 0.0269 & 0.0260 & 0.0287 & 0.0281 \\
$(150)$ & $R^{2}$ & -0.0222 & 0.0066 & 0.1532 & 0.3736 & 0.2394 & 0.2847 & 0.1594 & 0.1985 \\
\hline Nov 4, 2004- & MAE & 0.0043 & 0.0045 & 0.0083 & 0.0079 & 0.0116 & 0.0110 & 0.0112 & 0.0105 \\
Mar 30, 2005 & RMSE & 0.0060 & 0.0057 & 0.0118 & 0.0096 & 0.0141 & 0.0136 & 0.0136 & 0.0157 \\
$(105)$ & $R^{2}$ & -0.7723 & -0.6926 & -4.7860 & -3.0637 & -7.8151 & -7.372 & -7.1741 & -9.7939 \\
\hline Jan 6, 2005- & MAE & 0.0038 & 0.0040 & 0.00903 & 0.0072 & 0.0102 & 0.0094 & 0.0096 & 0.0101 \\
Mar 30, 2005 & RMSE & 0.0047 & 0.0046 & 0.01267 & 0.0087 & 0.0123 & 0.0113 & 0.0111 & 0.0149 \\
(60) & $R^{2}$ & -1.3531 & -2.0029 & -23.945 & -7.4391 & -17.9586 & -17.957 & -13.918 & -30.128 \\
\hline
\end{tabular}

market as another possible factor to explain the behavior of the overnight rate. This study has been carried out by imposing a certain dynamics to the jump intensity. It holds that the overreaction level is approximately twice as big as the "continuation" level.

The first two models implemented here are based on extensions of the well-known Poisson-Gaussian-ARCH structure. The difference between the two candidates can be found in the dynamics of the jump intensity $\lambda_{t}$. Our first approach consists of a piecewise function where $\lambda_{t}$ captures the three effects mentioned before. The second model exhibits a really time-varying pattern for $\lambda_{t}$, in concrete, the autoregressive conditional jump 
intensity (ARJI) by Chan and Maheu (2002) which also includes both the end of the maintenance period and the calendar effects since these effects behave like seasonal components. We conclude that both models drive to the same conclusions though the last one makes a better performance. Under the ARJI dynamics, we obtain daily time-varying ex-post probabilities which can be used to understand better the reaction of EONIA around the ECB's meetings.

Finally, we develop a two regime-switching model where each state is driven by an ARCH-Poisson-Gaussian under different specifications for the transition probabilities. The empirical results indicate that there is one regime characterised by high volatility and no reversion to the official rate and the other by low volatility and mean reversion. This evidence is agreed with other works such as Gray (1996) and so forth in the sense that the mean reversion does not always hold, that is why two regimes might result to be more appropriate than a single regime process to model interest rates. Moreover, two regime models outperform single regime ones in most cases in our out-of-sample forecasting analysis. According to our more generalized structure for the transition probability (a timevarying function depending on the size of the spread - the difference in absolute value between the official rate and the EONIA -, the end of the maintenance period and the calendar effects), it is shown that the probability of staying or switching to the regime of high volatility/no mean reversion is higher when any of these two seasonal effects happen. This might be a possible reason why there is more volatility in this period of no reversion since these seasonal components, which tend to concentrate here, lead to more volatility as we showed in other sections before. This regime is also characterised by a higher probability of occurring jumps and higher levels of interest rates. 


\section{Appendix}

\section{A.1) Likelihood function}

The density of $\Delta r_{t}$ in each state is:

$$
f\left(\Delta r_{t} \mid S_{t}, \Phi_{t-1}\right)=\left(1-\lambda_{S_{t}}\right) \times f\left(\Delta r_{t} \mid S_{t}, \Delta n_{t}=0, \Phi_{t-1}\right)+\lambda_{S_{t}} \times f\left(\Delta r_{t} \mid S_{t}, \Delta n_{t}=1, \Phi_{t-1}\right)
$$

such that

$$
f\left(\Delta r_{t} \mid S_{t}, \Delta n_{t}=j, \Phi_{t-1}\right)=\frac{1}{\sqrt{2 \pi\left(\sigma_{S_{t}, t}^{2}+j \psi_{S_{t}}^{2}\right)}} \exp \left(-\frac{\left(\Delta r_{t}-\mu_{S_{t}, t}-j \theta_{t}\right)^{2}}{2\left(\sigma_{S_{t}, t}^{2}+j \psi_{S_{t}}^{2}\right)}\right) .
$$

Finally, the conditional density of $\Delta r_{t}$ for this model is:

$$
f\left(\Delta r_{t} \mid \Phi_{t-1}\right)=p_{0, t} \times f\left(\Delta r_{t} \mid S_{t}=0, \Phi_{t-1}\right)+p_{1, t} \times f\left(\Delta r_{t} \mid S_{t}=1, \Phi_{t-1}\right)
$$

where $p_{S_{t}, t} \equiv P\left[S_{t}=i \mid \Phi_{t-1}\right]$ for $i=0,1$ such that

$$
\begin{aligned}
& p_{0, t}=p_{00} \times P\left[S_{t-1}=0 \mid \Phi_{t-1}\right]+\left(1-p_{11}\right) \times P\left[S_{t-1}=1 \mid \Phi_{t-1}\right] \\
& p_{1, t}=1-p_{0, t}
\end{aligned}
$$

where both $P\left[S_{t-1}=0 \mid \Phi_{t-1}\right]$ and $P\left[S_{t-1}=1 \mid \Phi_{t-1}\right]$ denote the ex-post or filtered probabilities from the previous period. The filtered probabilities follow Bayes' Theorem:

$$
P\left[S_{t}=0 \mid \Phi_{t}\right]=\frac{P\left[S_{t}=0 \mid \Phi_{t-1}\right] \times f\left(\Delta r_{t} \mid S_{t}=0, \Phi_{t-1}\right)}{f\left(\Delta r_{t} \mid \Phi_{t-1}\right)} ; \quad P\left[S_{t}=1 \mid \Phi_{t}\right]=1-P\left[S_{t}=0 \mid \Phi_{t}\right]
$$


Finally, the log-likelihood is obtained as $\sum_{t=1}^{T} \ln f\left(\Delta r_{t} \mid \Phi_{t-1}\right)$ where $T$ is the sample size.

\section{A.2) Smoothed probabilities}

The smoothed probabilities $P\left[S_{t} \mid \Phi_{T}\right]$, where $t=1,2, \ldots, T$, are also of interest in determining if and when regime switches occur. These are obtained by the smoothing algorithm in Kim and Nelson (1999):

$$
P\left[S_{t}=i \mid \Phi_{T}\right]=\sum_{j=0}^{1} P\left[S_{t}=i, S_{t+1}=j \mid \Phi_{T}\right]
$$

where

$$
P\left[S_{t}=i, S_{t+1}=j \mid \Phi_{T}\right]=\frac{P\left[S_{t+1}=j \mid \Phi_{T}\right] \times P\left[S_{t}=i \mid \Phi_{t}\right] \times P\left[S_{t+1}=j \mid S_{t}=i\right]}{P\left[S_{t+1}=j \mid \Phi_{t}\right]} .
$$




\section{References}

Ang, A. and G. Bekaert (2002a): "Regime switches in interest rates", Journal of Business and Economic Statistics, 20:2, 163-182.

Ang, A. and G. Bekaert (2002b): "Short rate nonlinearities and regime switches", Journal of Economic Dynamics and Control, 26, 1243-1274.

Angeloni, I. and E. Bisagni (2002): "Liquidity effects in the euro area", mimeo, July 2002, ECB and University of California, San Diego.

Balduzzi, P., Bertola, G. and S. Foresi, (1997): "A model of target changes and the term structure of interest rates”, Journal of Monetary Economics, 39, 223-249.

Bartolini, L., Bertola, G. and A. Prati (2002): "Day-to-day monetary policy and the volatility of the federal funds rate", Journal of Money, Credit, and Banking, 34, 137-159.

Bindseil, U. and F. Seitz (2001): "The supply and demand for Eurosystem deposits the first 18 months", ECB Working Paper No. 44.

Blanco, R. (2005): "Is the volatility of the EONIA transmitted to longer term euro money market interest rate?", Working Paper, Bank of Spain.

Cai, J. (1994): "A Markov model of unconditional variance in ARCH", Journal of Business and Economic Statistics 12, 309-316.

Cassola, N. and C. Morana (2003): "Volatility of interest rates in the euro area: evidence from high frequency data", ECB Working Paper No. 235.

Cassola, N. and C. Morana (2004): "Transmission of volatility in the euro area money market", ECB, mimeo.

Chan, W.H. and J.M. Maheu (2002): "Conditional jump dynamics in stock market returns", Journal of Business and Economics Statistics, 20:3, 377-389.

Dahlquist, M. and S.F. Gray (2000): "Regime-switching and interest rates in the European monetary system", Journal of International Economics, 50, 399-419.

Das, S., (2002): “The surprise element: jumps in interest rates", Journal of Econometrics $106,27-65$.

Gaspar, V., Perez Quirós, G. and J. Sicilia (2001): “The ECB monetary policy strategy and the money markets", International Journal of Finance and Economics 6 (4), 325342.

Gray, S.F. (1996): "Modeling the conditional distribution of interest rates as a regimeswitching process", Journal of Financial Economics 42, 27-62. 
Guan, L.K., Ting, C. and M. Warachka (2005): "The implied jump risk of LIBOR rates", Journal of Banking and Finance, forthcoming.

Hamilton, J. D. and R. Susmel (1994): “Autoregressive conditional heteroscedasticity and changes in regime", Journal of Econometrics 64, 307-333.

Hamilton, J. D. (1996): "The daily market for fed funds", Journal of Political Economy 104 (1), 26-56.

Hamilton, J. D. and O. Jordá (2002): “A model for the federal funds rate target", Journal of Political Economy 110, 1135-1167.

Hartmann, P., Manna, M. and A. Manzanares (2001): "The microstructure of the Euro money market", Journal of International Money and Finance 20, 895-948.

Kim, C. and C.R. Nelson (1999): State-space models with regime switching, MIT Press.

Mankiw, N.G. and J.A. Miron (1986): "The changing behavior of the term structure of interest rates", Quarterly Journal of Economics 101, 211-228.

Manna, M., Pill, H. and G. Quirós (2001): “The Eurosystem's operational framework in the context of the ECB's monetary policy strategy", European Central Bank, mimeo.

Maheu, J.M. and T.M. McCurdy (2004): "News arrival, jump dynamics, and volatility components for individual stock returns", Journal of Finance, 59:2, 755-793.

Moschitz, J. (2004a): "The determinants of the overnight interest rate in the euro area", ECB Working Paper No. 393.

Moschitz, J. (2004b): "Monetary policy implementation and volatility in the Euro area money market", Working Paper, Universitat Autònoma de Barcelona.

Pérez Quirós, G. and H., Rodríguez Mendizábal (2005): “The daily market for funds in Europe: What has changed with the EMU", Journal of Money, Credit and Banking, forthcoming.

Prati, A., Bartolini, L. and G. Bertola (2002): "The overnight interbank market: Evidence from the G-7 and the Euro Zone", Journal of Banking \& Finance 27, 2045-2083

Sarno, L., Thornton, D.L. and G. Valente (2005): "Federal funds rate prediction", Journal of Money, Credit and Banking, forthcoming.

Würtz, F.R. (2003): “A comprehensive model on the Euro overnight rate”, ECB Working Paper 207. 